Groupe d'Annecy

Groupe de Lyon

Laboratoire

d'Annecy-le-Vieux de

Physique des Particules

\author{
Ecole Normale
}

Supérieure de Lyon

\title{
Remarks On Quantum Integration
}

\author{
Chryssomalis Chryssomalakos* \\ Laboratoire de Physique Théorique ENSLAPP耳 \\ Chemin de Bellevue BP 110, F-74941 Annecy-le-Vieux Cedex, France.
}

\begin{abstract}
We give a general integration prescription for finite dimensional braided Hopf algebras, deriving the N-dimensional quantum superplane integral as an example. The transformation properties of the integral on the quantum plane are found. We also discuss integration on quantum group modules that lack a Hopf structure.
\end{abstract}

ENSLAPP-A-562/95

December 1995

\footnotetext{
${ }^{1}$ URA 14-36 du CNRS, associée à l'Ecole Normale Supérieure de Lyon et à l'Université de Savoie.

${ }^{*}$ chryss@lapp.in2p3.fr
} 


\section{Introduction}

The emergence of Hopf algebras, during the last decade, as a promising framework within which new physical symmetries can be accomodated, has prompted an interest in the theory and techniques of integration on them. Similar remarks hold for braided Hopf algebras which, more recently, have provided a still further extension of the classical concept of a group, marrying quantization with nontrivial statistics.

Integrals on (finite dimensional) Hopf algebras have been studied extensively, see for example [12, 19] and references therein. For the braided case see the treatment in [13, 14 for the basics of the theory - some examples appear in [3, 10]. From the point of view of a physicist who is interested in the basic elements of the theory and in applications, the situation presernts certain problems. The results are generally scattered and when they do become available, the disparity of the methods employed in them prohibits the formation of a clear image of the minimum background required to explore the field. When it comes to applications, results are extracted (often ingeniously) from particular properties of individual examples - no general integration prescription seems to be available for the quantum space wanderers. The closest one can come to such a prescription in the literature is perhaps the trace formula of Radford and Larson [11] (and a braided analogue of it in [18]) which, however, occasionally returns trivial (i.e. identically zero) results (there doesn't seem to exist a description of when exactly it fails either). Our purpose in this paper is thus basically twofold. On the one hand, we aim at providing simple, self contained proofs of basic results, using only the Hopf algebra axioms we assume the reader to be familiar with, attempting in this way a demonstration of what can be accomplished with a rather minimal set of tools. On the other hand, addressing the problem of the missing integration prescription, we give an explicit formula for the integral on any finite dimensional braided Hopf algebra (FDBHA) and show that it is always nontrivial, commenting along the way on the conditions under which the trace formula fails.

The paper is structured as follows: in section 2 we present the notation we use and collect some basic formulas we need in subsequent proofs. Section 3 starts with background information on integrals on (nonbraided) Hopf algebras. We then give a modified trace formula for the integral and prove its nontriviality. We also introduce a "vacuum expectation value" approach to integration, discuss properties of right Fourier transforms and prove a number of useful formulas. Section 4 supplies the braided version of the modified trace formula and the vacuum projectors and discusses, as an example, the integral on the N-dimensional quantum superplane. Also included are some comments on the transformation properties of the integral on the N-dimensional quantum plane. The last section provides an integration prescription (with some modest assumptions) for quantum group modules that lack a braided Hopf structure.

\section{Hopf Algebras}

The language used in the following is predominantly that of Hopf algebras - we refer the reader to [1, 15, 22] for an introduction to the subject. Concerning the notation, we denote by $\Delta, \epsilon, S$ the coproduct, counit and antipode respectively and by $\Delta_{\mathcal{A}}, \mathcal{u} \Delta$ the right $\mathcal{A}$ and left $\mathcal{U}$-coactions respectively. Sweedler-like conventions are employed throughout - thus $\Delta a=a_{(1)} \otimes a_{(2)}$, $(\Delta \otimes \mathrm{id}) \circ \Delta(a)=a_{(1)} \otimes a_{(2)} \otimes a_{(3)}$ etc. . Also, $\Delta_{\mathcal{A}}(x)=x^{(1)} \otimes x^{\left(2^{\prime}\right)}, \mathcal{u} \Delta(a)=a^{(\overline{1})} \otimes a^{(2)}$ and, for example, $(\mathrm{id} \otimes \Delta) \circ \Delta_{\mathcal{A}}(x)=x^{(1)} \otimes x^{\left(2^{\prime}\right)}{ }_{(1)} \otimes x^{\left(2^{\prime}\right)}{ }_{(2)}=x^{(1)} \otimes x^{\left(2^{\prime}\right)} \otimes x^{\left(3^{\prime}\right)}$ and so on. By $\mathcal{A}$ we 
will generally denote a function type Hopf algebra (its elements will be denoted by $a, b$, etc.) - $\mathcal{U}$ will stand for its dual Hopf algebra (universal enveloping algebra type) with elements $x, y$ etc. . The duality is via a nondegenerate inner product $\langle\cdot, \cdot\rangle$ that relates the algebra structure in $\mathcal{A}$ with the coalgebra stucture in $\mathcal{U}$ and vice-versa. The universal $R$-matrix is denoted by $\mathcal{R}=\mathcal{R}^{(1)} \otimes \mathcal{R}^{(2)} ; \mathcal{R}^{\prime}$ stands for $\tau(\mathcal{R})$ with $\tau(a \otimes b)=b \otimes a$. $\mathcal{R}$ satisfies

$$
\Delta^{\prime}(x)=\mathcal{R} \Delta(x) \mathcal{R}^{-1} \quad \forall x \in \mathcal{U}
$$

as well as

$$
\begin{aligned}
(\Delta \otimes \mathrm{id}) \mathcal{R} & =\mathcal{R}^{13} \mathcal{R}^{23} \\
(\mathrm{id} \otimes \Delta) \mathcal{R} & =\mathcal{R}^{13} \mathcal{R}^{12}
\end{aligned}
$$

(with $\mathcal{R}^{13} \equiv \mathcal{R}^{(1)} \otimes 1_{\mathcal{U}} \otimes \mathcal{R}^{(2)}$ etc.)

Given a pair of dual Hopf algebras, one can construct their semidirect product $\mathcal{A} \rtimes \mathcal{U}$ with $\mathcal{A}, \mathcal{U}$ trivially embedded in it and cross relations

$$
x a=a_{(1)}\left\langle x_{(1)}, a_{(2)}\right\rangle x_{(2)}, \quad a x=x_{(2)}\left\langle x_{(1)}, S^{-1}\left(a_{(2)}\right)\right\rangle a_{(1)} .
$$

The above commutation relations guarrantee that [24]

$$
\mathcal{A} \otimes \mathcal{U} \ni a \otimes x \neq 0 \Rightarrow x a \neq 0
$$

with $x a \in \mathcal{A} \rtimes \mathcal{U}$. The action $\triangleright$ of $\mathcal{U}$ on $\mathcal{A}$ is given by $x \triangleright a=a_{(1)}\left\langle x, a_{(2)}\right\rangle$. The same symbol will denote the (adjoint) action of $\mathcal{U}$ on $\mathcal{U}: x \triangleright y=x_{(1)} y S\left(x_{(2)}\right)=y^{(1)}\left\langle x, y^{\left(2^{\prime}\right)}\right\rangle$. The canonical element in $\mathcal{U} \otimes \mathcal{A}$ is written like $C=e_{i} \otimes f^{i}$ with $\left\{e_{i}\right\},\left\{f^{i}\right\}$ dual (in the sense that $\left\langle e_{i}, f^{j}\right\rangle=\delta_{i}^{j}$ ) linear bases in $\mathcal{U}, \mathcal{A}$ respectively. It holds

$$
\begin{aligned}
(\Delta \otimes \mathrm{id}) C_{12} & =C_{13} C_{23} \\
(\mathrm{id} \otimes \Delta) C_{12} & =C_{12} C_{13} \\
(\epsilon \otimes \mathrm{id}) C & =1 \\
(\mathrm{id} \otimes \epsilon) C & =1 \\
(S \otimes \mathrm{id}) C & =C^{-1} \\
(\mathrm{id} \otimes S) C & =C^{-1},
\end{aligned}
$$

as well as

$$
\begin{aligned}
\Delta_{\mathcal{A}}(a) \equiv \Delta(a) & =C(a \otimes 1) C^{-1} \\
\Delta_{\mathcal{A}}(x) & =C(x \otimes 1) C^{-1} \\
\mathcal{u} \Delta(a) & =C^{-1}(1 \otimes a) C \\
\mathcal{u} \Delta(x) \equiv \Delta(x) & =C^{-1}(1 \otimes x) C .
\end{aligned}
$$

Either of (4) encodes the information about the inner product $\langle x, a\rangle$. To make this precise, we introduce $\mathcal{U}$ and $\mathcal{A}$-right vacua, denoted by $\left|\Omega_{\mathcal{U}}\right\rangle$ and $\left|\Omega_{\mathcal{A}}\right\rangle$ respectively, which satisfy [3]

$$
\begin{aligned}
x\left|\Omega_{\mathcal{U}}\right\rangle & =\epsilon(x)\left|\Omega_{\mathcal{U}}\right\rangle \\
a\left|\Omega_{\mathcal{A}}\right\rangle & =\epsilon(a)\left|\Omega_{\mathcal{A}}\right\rangle .
\end{aligned}
$$


Left vacua $\left\langle\Omega_{\mathcal{U}}\right|,\left\langle\Omega_{\mathcal{A}}\right|$ are defined analogously. In terms of these, the inner product $\langle x, a\rangle$ can be given as the "expectation value"

$$
\begin{aligned}
\left\langle\Omega_{\mathcal{A}}|x a| \Omega_{\mathcal{U}}\right\rangle & =\left\langle\Omega_{\mathcal{A}}\left|a_{(1)}\left\langle x_{(1)}, a_{(2)}\right\rangle x_{(2)}\right| \Omega_{\mathcal{U}}\right\rangle \\
& =\langle x, a\rangle
\end{aligned}
$$

if we normalize the vacua so that $\left\langle\Omega_{\mathcal{A}} \mid \Omega_{\mathcal{U}}\right\rangle=\left\langle\Omega_{\mathcal{U}} \mid \Omega_{\mathcal{A}}\right\rangle=1$. Similarly, the adjoint action of $\mathcal{U}$ on $\mathcal{A}$ can be written as

$$
x a\left|\Omega_{\mathcal{U}}\right\rangle=x \triangleright a\left|\Omega_{\mathcal{U}}\right\rangle .
$$

\section{$3 \quad$ Integration on Hopf Algebras}

\subsection{Background}

We list here known results about invariant integrals on Hopf algebras that we will use later - more details can be found in [22, [1]. Some of the proofs are also supplied in order to familiarize the reader with the usage of the tools presented in section 2. To prevent potential divergence problems from distracting the formulation of concepts, we deal throughout with finite dimensional Hopf algebras - some of the results though retain their validity in the infinite dimensional case as well.

We start with two dually paired Hopf algebras $\mathcal{U}, \mathcal{A}$ with (finite) dual bases $\left\{e_{i}\right\},\left\{f^{j}\right\}$ respectively. We define a right (invariant) integral in $\mathcal{A}$ as a map $\langle\cdot\rangle^{R}: \mathcal{A} \rightarrow k$ with the property

$$
\left\langle a_{(1)}\right\rangle^{R} a_{(2)}=\langle a\rangle^{R} 1_{\mathcal{A}}
$$

for all $a$ in $\mathcal{A}$. We call $\langle\cdot\rangle^{R}$ trivial if all $\left\langle f^{i}\right\rangle^{R}$ are zero. Left (invariant) integrals are similarly defined via

$$
a_{(1)}\left\langle a_{(2)}\right\rangle^{L}=1_{\mathcal{A}}\langle a\rangle^{L} .
$$

As we shall soon see, when $\left\langle 1_{\mathcal{A}}\right\rangle^{R} \neq 0$ (or $\left\langle 1_{\mathcal{A}}\right\rangle^{L} \neq 0$ ), left and right integrals are proportional and can therefore be normalized so that they coincide. One can now introduce the element $\delta_{\mathcal{U}}^{R} \in \mathcal{U}$ (the right delta function in $\mathcal{U}$ ) which implements the right integral in $\mathcal{A}$ via

$$
\left\langle\delta_{\mathcal{U}}^{R}, a\right\rangle=\langle a\rangle^{R}
$$

(so that $\left.\epsilon\left(\delta_{\mathcal{U}}^{R}\right)=\left\langle 1_{\mathcal{A}}\right\rangle^{R}\right)$. This allows us to write

$$
\delta_{\mathcal{U}}^{R}=\left\langle f^{i}\right\rangle^{R} e_{i}
$$

(in the mathematics literature $\delta_{\mathcal{U}}^{R}$ is often called a right integral in $\mathcal{U}$ - we will not use this terminology here). For arbitrary $a$ in $\mathcal{A}$ we have

$$
\begin{aligned}
\left\langle\delta_{\mathcal{U}}^{R} x, a\right\rangle & =\left\langle\delta_{\mathcal{U}}^{R}, a_{(1)}\right\rangle\left\langle x, a_{(2)}\right\rangle \\
& =\left\langle a_{(1)}\right\rangle^{R}\left\langle x, a_{(2)}\right\rangle \\
& =\langle a\rangle^{R}\left\langle x, 1_{\mathcal{A}}\right\rangle \\
& =\epsilon(x)\langle a\rangle^{R} \\
& =\left\langle\delta_{\mathcal{U}}^{R} \epsilon(x), a\right\rangle
\end{aligned}
$$


therefore

$$
\delta_{\mathcal{U}}^{R} x=\delta_{\mathcal{U}}^{R} \epsilon(x) \quad \forall x \in \mathcal{U} .
$$

Taking antipodes in the above equation we find $(\epsilon(x)=\epsilon(S(x)))$

$$
S(x) S\left(\delta_{\mathcal{U}}^{R}\right)=\epsilon(S(x)) S\left(\delta_{\mathcal{U}}^{R}\right)
$$

which, for invertible $S$ gives

$$
x S\left(\delta_{\mathcal{U}}^{R}\right)=\epsilon(x) S\left(\delta_{\mathcal{U}}^{R}\right) \quad \forall x \in \mathcal{U},
$$

in other words, $S\left(\delta_{\mathcal{U}}^{R}\right)$ implements a left integral and we can therefore take $S\left(\delta_{\mathcal{U}}^{R}\right)=\delta_{\mathcal{U}}^{L}$. Since $\epsilon(S(x))=\epsilon(x)$, we find that $\left\langle 1_{\mathcal{A}}\right\rangle^{R}=\left\langle 1_{\mathcal{A}}\right\rangle^{L} \equiv\left\langle 1_{\mathcal{A}}\right\rangle(\langle\cdot\rangle$ denotes a biinvariant integral). Consider now the product $\delta_{\mathcal{U}}^{R} S\left(\delta_{\mathcal{U}}^{R}\right)$. We have

$$
\delta_{\mathcal{U}}^{R} S\left(\delta_{\mathcal{U}}^{R}\right)=\delta_{\mathcal{U}}^{R} \epsilon\left(S\left(\delta_{\mathcal{U}}^{R}\right)\right)=\delta_{\mathcal{U}}^{R} \epsilon\left(\delta_{\mathcal{U}}^{R}\right)=\delta_{\mathcal{U}}^{R}\left\langle 1_{\mathcal{A}}\right\rangle
$$

and also

$$
\delta_{\mathcal{U}}^{R} S\left(\delta_{\mathcal{U}}^{R}\right)=\epsilon\left(\delta_{\mathcal{U}}^{R}\right) S\left(\delta_{\mathcal{U}}^{R}\right)=\left\langle 1_{\mathcal{A}}\right\rangle S\left(\delta_{\mathcal{U}}^{R}\right)
$$

therefore, in the unimodular case where $\left\langle 1_{\mathcal{A}}\right\rangle \neq 0$ (so that we can normalize $\left\langle 1_{\mathcal{A}}\right\rangle=1$ ), it holds

$$
\begin{aligned}
\delta_{\mathcal{U}}^{R} & =S\left(\delta_{\mathcal{U}}^{R}\right) \\
x \delta_{\mathcal{U}}^{R} & =\epsilon(x) \delta_{\mathcal{U}}^{R} \quad \forall x \in \mathcal{U} \\
\langle a\rangle & =\langle S(a)\rangle \quad \forall a \in \mathcal{A} .
\end{aligned}
$$

Concerning uniqueness, assume that a second right integral $\langle\cdot\rangle^{R^{\prime}}$ exists and let $\delta_{\mathcal{U}^{\prime}}^{R^{\prime}}$ be the element of $\mathcal{U}$ that implements it. We then get $\delta_{\mathcal{U}}^{R} S\left(\delta_{\mathcal{U}}^{R^{\prime}}\right)=\left\langle 1_{\mathcal{A}}\right\rangle S\left(\delta_{\mathcal{U}}^{R^{\prime}}\right)$ and $\delta_{\mathcal{U}}^{R} S\left(\delta_{\mathcal{U}}^{R^{\prime}}\right)=\delta_{\mathcal{U}}^{R}\left\langle 1_{\mathcal{A}}\right\rangle^{\prime}$. For $\left\langle 1_{\mathcal{A}}\right\rangle=1$ we conclude that $\left\langle 1_{\mathcal{A}}\right\rangle^{\prime}=1$ implies $\delta_{\mathcal{U}}^{R}=S\left(\delta_{\mathcal{U}}^{R^{\prime}}\right)=\delta_{\mathcal{U}}^{R^{\prime}}$ while $\left\langle 1_{\mathcal{A}}\right\rangle^{\prime}=0$ implies that $\langle\cdot\rangle^{\prime}$ is trivial.

Radford and Larson [11] have shown that

$$
\langle a\rangle_{t r}^{R} \equiv\left\langle S^{2}\left(e_{i}\right), f^{i} a\right\rangle
$$

defines a right integral on $\mathcal{A}$ which though, in some cases (as [11] warns), is trivial. It is this shortcoming of (20) that (among other things) motivated our formula for the integral of the next section, which is shown to be nontrivial for any FDHA. One can derive from (20) that $\left\langle S^{-2}\left(e_{i}\right), f^{i} a\right\rangle,\left\langle S^{-2}\left(e_{i}\right), a f^{i}\right\rangle$ define left and right (again, in some cases trivial) integrals respectively. For $\sigma \equiv\left\langle S^{2}\left(e_{i}\right), f^{i}\right\rangle \neq 0$ and $\sigma^{\prime} \equiv\left\langle S^{-2}\left(e_{i}\right), f^{i}\right\rangle$ we conclude

$$
\left\langle S^{-2}\left(e_{i}\right), f^{i} a\right\rangle=\left\langle S^{-2}\left(e_{i}\right), a f^{i}\right\rangle=\frac{\sigma^{\prime}}{\sigma}\langle a\rangle_{t r}^{R} .
$$

We summarize the main points: when $\left\langle 1_{\mathcal{A}}\right\rangle=1,\langle\cdot\rangle$ is biinvariant, unique and (19) holds. (20) defines a (sometimes trivial) right integral and when $\sigma \neq 0$, (21) holds. Considerably more is known about integrals on Hopf algebras - in the interest of self containment we have only mentioned above what we can prove here. 


\subsection{A modified trace formula}

We want to present now a modified version of the trace formula (20) that overcomes the limitations mentioned above. It is given by

$$
\langle a\rangle^{R} \delta_{\mathcal{A}}^{R}=\left\langle e_{j} S^{-2}\left(e_{i}\right), a f^{i}\right\rangle f^{j}
$$

(for the remainder of this paper, $\langle a\rangle^{R} \delta_{\mathcal{A}}^{R}$ is defined by the rhs of (22)). Notice that (22) also defines (for some nonzero $\langle a\rangle^{R}$ which we can normalize to 1) the right delta function in $\mathcal{A}$. By pairing both sides of (22) with $x$ in $\mathcal{U}$ we conclude

$$
\langle x\rangle^{R}\langle a\rangle^{R}=\left\langle x S^{-2}\left(e_{i}\right), a f^{i}\right\rangle
$$

The proof of invariance is quite analogous to that of (20). What is interesting is the following

Lemma 1 The integral $\langle\cdot\rangle^{R}$ defined by (29) is nontrivial for any FDHA $\mathcal{A}$.

Proof: Set $\Theta_{l}^{k} \equiv\left\langle e_{l} S^{-2}\left(e_{i}\right), f^{k} f^{i}\right\rangle$ and compute

$$
\begin{aligned}
\mathcal{A} \rtimes \mathcal{U} \ni S^{-2}\left(e_{i}\right) f^{i} & =f_{(1)}^{i}\left\langle S^{-2}\left(e_{i_{(1)}}\right), f_{(2)}^{i}\right\rangle S^{-2}\left(e_{i_{(2)}}\right) \\
& =f_{(1)}^{i} f_{(1)}^{j}\left\langle S^{-2}\left(e_{i}\right), f_{(2)}^{i} f_{(2)}^{j}\right\rangle S^{-2}\left(e_{j}\right) \\
& =S^{-2}\left(f^{i}\right) f^{j}\left\langle e_{i} S^{-2}\left(e_{k}\right), f^{k} f^{l}\right\rangle S^{-2}\left(e_{j}\right) S^{-2}\left(e_{l}\right) \\
& =\Theta_{i}^{l} S^{-2}\left(f^{i}\right) f^{j} S^{-2}\left(e_{j}\right) S^{-2}\left(e_{l}\right) .
\end{aligned}
$$

We now employ (5) to conclude that not all $\Theta_{i}^{l}$ are zero $\left(\right.$ since $S^{-2}\left(e_{i}\right) \otimes f^{i} \neq 0$ in $\mathcal{U} \otimes \mathcal{A}$ ). Alternatively, one can compute directly the integral [23]

$$
\begin{aligned}
\left\langle S^{-1}\left(\delta_{\mathcal{A}}^{R}\right)\right\rangle^{R} & =\left\langle e_{i}\right\rangle^{R}\left\langle S^{-1}\left(f^{i}\right)\right\rangle^{R} \\
& =\left\langle e_{i} e_{j}, S^{-1}\left(f^{i}\right) S^{-2}\left(f^{j}\right)\right\rangle \\
& =\left\langle e_{i}, S^{-1}\left(f_{(1)}^{i}\right) S^{-2}\left(f_{(2)}^{i}\right)\right\rangle \\
& =\left\langle e_{i}, \epsilon\left(f^{i}\right) 1_{\mathcal{A}}\right\rangle \\
& =\left\langle 1_{\mathcal{U}}, 1_{\mathcal{A}}\right\rangle \\
& =1,
\end{aligned}
$$

which shows nontriviality in both $\mathcal{U}$ and $\mathcal{A}$ (of course, $\left\langle e_{i}\right\rangle^{R}\left\langle S^{-1}\left(f^{i}\right)\right\rangle^{R}=1$ is a stronger statement).

Defining $\Theta=\Theta^{(1)} \otimes \Theta^{(2)}=\Theta_{k}^{l} f^{k} \otimes e_{l}$ we get

$$
\langle a\rangle^{R} \delta_{\mathcal{A}}^{R}=\Theta^{(1)}\left\langle\Theta^{(2)}, a\right\rangle \quad\langle x\rangle^{R} \delta_{\mathcal{U}}^{R}=\left\langle x, \Theta^{(1)}\right\rangle \Theta^{(2)} .
$$

Related to the above proof is

Lemma 2 For $\mathcal{A}$ a FDHA, it holds (a in $\mathcal{A})$

$$
\left\langle a f^{i}\right\rangle^{R}=0 \quad \forall i \Rightarrow a=0
$$


Proof: Assuming $\left\langle a f^{i}\right\rangle^{R}=0$ for all $i$ we get

$$
\begin{aligned}
0 & =\left\langle S^{-1}\left(e_{i}\right)\right\rangle^{R}\left\langle a f_{(1)}^{i}\right\rangle^{R} S\left(f_{(2)}^{i}\right) \\
& =\left\langle S^{-1}\left(e_{i}\right)\right\rangle^{R}\left\langle a_{(1)} f_{(1)}^{i}\right\rangle^{R} a_{(2)} f_{(2)}^{i} S\left(f_{(3)}^{i}\right) \\
& =\left\langle S^{-1}\left(e_{i}\right)\right\rangle^{R}\left\langle a_{(1)} f^{i}\right\rangle^{R} a_{(2)} \\
& =\left\langle S^{-1}\left(e_{i}\right)\right\rangle^{R}\left\langle f^{i}\right\rangle^{R} a \\
& =a
\end{aligned}
$$

where, in the last line, use was made of (25)).

There exist formulas similar to (23) for other combinations of invariance properties

$$
\begin{aligned}
\langle x\rangle^{L}\langle a\rangle^{L} & \sim\left\langle e_{i} x, S^{-2}\left(f^{i}\right) a\right\rangle \\
\langle x\rangle^{L}\langle a\rangle^{R} & \sim\left\langle x S^{2}\left(e_{i}\right), f^{i} a\right\rangle \\
\langle x\rangle^{R}\langle a\rangle^{L} & \sim\left\langle e_{i} x, a S^{2}\left(f^{i}\right)\right\rangle .
\end{aligned}
$$

We could have used any of these formulas as our basic definition of the integral. When we deal with braided Hopf algebras we use in fact the analogue of the second of (28) as our starting point. The coefficient of proportionality in the above formulas depends on which function's integral we normalize to 1 . The relation with the trace formula (20) is illuminated by the following

Lemma 3 For $\mathcal{A}, \mathcal{U}$ dually paired FDHAs, it holds ( $a$ in $\mathcal{A}, x$ in $\mathcal{U}$ )

$$
\langle x\rangle_{t r}^{R}\langle a\rangle_{t r}^{R}=\sigma\left\langle x e_{i}, S^{2}\left(f^{i}\right) a\right\rangle .
$$

Proof:

$$
\begin{aligned}
\langle x\rangle_{t r}^{R}\langle a\rangle_{t r}^{R} & =\left\langle e_{i} x, S^{2}\left(f^{i}\right)\right\rangle\left\langle e_{k}, S^{2}\left(f^{k}\right) a\right\rangle \\
& =\left\langle e_{i} x_{(1)}, S^{2}\left(f^{i}\right)\right\rangle\left\langle x_{(2)} e_{k}, S^{2}\left(f^{k}\right) a\right\rangle \\
& =\left\langle e_{i}, S^{2}\left(f_{(1)}^{i}\right)\right\rangle\left\langle x_{(1)}, S^{2}\left(f_{(2)}^{i}\right)\right\rangle\left\langle x_{(2)} e_{k}, S^{2}\left(f^{k}\right) a\right\rangle \\
& =\left\langle e_{i}, S^{2}\left(f_{(1)}^{i}\right)\right\rangle\left\langle x_{(1)} e_{k(2)} S^{-1}\left(e_{k(1)}\right), S^{2}\left(f_{(2)}^{i}\right)\right\rangle\left\langle x_{(2)} e_{k(3)}, S^{2}\left(f^{k}\right) a\right\rangle \\
& =\left\langle e_{i}, S^{2}\left(f_{(1)}^{i}\right)\right\rangle\left\langle S^{-1}\left(e_{k(1)}\right), S^{2}\left(f_{(3)}^{i}\right)\right\rangle\left\langle x e_{k(2)}, S^{2}\left(f_{(2)}^{i}\right) S^{2}\left(f^{k}\right) a\right\rangle \\
& =\left\langle e_{i} e_{j} e_{l}, S^{2}\left(f^{i}\right)\right\rangle\left\langle e_{k(1)}, S\left(f^{l}\right)\right\rangle\left\langle x e_{k(2)}, S^{2}\left(f^{j}\right) S^{2}\left(f^{k}\right) a\right\rangle \\
& \left.=\left\langle e_{i} e_{j} S\left(e_{k(1)}\right), S^{2}\left(f^{i}\right)\right\rangle\left\langle x e_{k(2)}, S^{2}\left(f^{j}\right)\right) S^{2}\left(f^{k}\right) a\right\rangle \\
& =\left\langle e_{i} e_{j(1)} S\left(e_{j(2)}\right), S^{2}\left(f^{i}\right)\right\rangle\left\langle x e_{j(3)}, S^{2}\left(f^{j}\right) a\right\rangle \\
& =\left\langle e_{i}, S^{2}\left(f^{i}\right)\right\rangle\left\langle x e_{j}, S^{2}\left(f^{j}\right) a\right\rangle .
\end{aligned}
$$

As a corollary, we infer that, when $\sigma=0,\langle\cdot\rangle_{t r}^{R}$ is trivial in $\mathcal{U}$ or $\mathcal{A}$ (or both); when $\sigma \neq 0$, it is nontrivial in both $\mathcal{U}$ and $\mathcal{A}$. 


\subsection{Vacuum Projectors}

We give here a formulation of invariant integration in which the integral of a function is regarded as its "vacuum expectation value". First, notice that right invariance can also be expressed as $(x$ in $\mathcal{U}, a$ in $\mathcal{A})$

$$
\langle x \triangleright a\rangle=\epsilon(x)\langle a\rangle .
$$

Recall now the $\mathcal{U}$ and $\mathcal{A}$-vacua introduced in section 2. We could define, in terms of these, our "vacuum" integral via an equation like $(a \in \mathcal{A})$ [26]

$$
\langle a\rangle_{v} \sim\left\langle\Omega_{\mathcal{U}}|a| \Omega_{\mathcal{U}}\right\rangle
$$

invariance in the form (30) is automatically satisfied. However, as we shall soon see, it is more natural, in this case, to work instead with quantities like $\left|\Omega_{\mathcal{A}}\right\rangle\left\langle\Omega_{\mathcal{A}}|,| \Omega_{\mathcal{A}}\right\rangle\left\langle\Omega_{\mathcal{U}}\right|$ etc., i.e. with operators rather than states. The reason is that the former can be realized in $\mathcal{A} \rtimes \mathcal{U}$ and hence their properties can be derived while those of the latter have to be introduced "by hand". We aim therefore at a definition like

$$
\langle a\rangle_{v} \sim\left|\Omega_{\mathcal{A}}\right\rangle\left\langle\Omega_{\mathcal{U}}|a| \Omega_{\mathcal{U}}\right\rangle\left\langle\Omega_{\mathcal{A}}\right|
$$

We expect the rhs of (32) to be proportional to $\delta_{\mathcal{A}}$ (at least, under certain conditions), consistent with its property to return counit when multiplied by functions either from the left or from the right. What we need next is to find quantities in $\mathcal{A} \rtimes \mathcal{U}$ that represent the operators $\left|\Omega_{\mathcal{A}}\right\rangle\left\langle\Omega_{\mathcal{U}}|,| \Omega_{\mathcal{U}}\right\rangle\left\langle\Omega_{\mathcal{A}}\right|$. We recall at this point a result of [2]: the vacuum projectors $E, \bar{E}$ defined by

$$
E=S^{-1}\left(f^{i}\right) e_{i}, \quad \bar{E}=S^{2}\left(e_{i}\right) f^{i}
$$

satisfy

$$
\begin{aligned}
E a & =S^{-1}\left(f^{i}\right) e_{i} a \\
& =S^{-1}\left(f^{i}\right) a_{(1)}\left\langle e_{i_{(1)}}, a_{(2)}\right\rangle e_{i_{(2)}} \\
& =S^{-1}\left(f^{j}\right) S^{-1}\left(f^{i}\right) a_{(1)}\left\langle e_{i}, a_{(2)}\right\rangle e_{j} \\
& =S^{-1}\left(f^{j}\right) S^{-1}\left(a_{(2)}\right) a_{(1)} e_{j} \\
& =\epsilon(a) E
\end{aligned}
$$

for all $a$ in $\mathcal{A}$, as well as

$$
\begin{aligned}
x E & =x S^{-1}\left(f^{i}\right) e_{i} \\
& =S^{-1}\left(f_{(2)}^{i}\right)\left\langle x_{(1)}, S^{-1}\left(f_{(1)}^{i}\right)\right\rangle x_{(2)} e_{i} \\
& =S^{-1}\left(f^{j}\right)\left\langle x_{(1)}, S^{-1}\left(f^{i}\right)\right\rangle x_{(2)} e_{i} e_{j} \\
& =S^{-1}\left(f^{j}\right) x_{(2)} S^{-1}\left(x_{(1)}\right) e_{j} \\
& =\epsilon(x) E
\end{aligned}
$$

for all $x$ in $\mathcal{U}$, while we can similarly show that

$$
\begin{array}{ll}
\bar{E} x=\epsilon(x) \bar{E} & \forall x \in \mathcal{U} \\
a \bar{E}=\epsilon(a) \bar{E} & \forall a \in \mathcal{A} .
\end{array}
$$


Furthermore, $E^{2}=E$ and $\bar{E}^{2}=\bar{E}$ which allows us to write $E=\left|\Omega_{\mathcal{U}}\right\rangle\left\langle\Omega_{\mathcal{A}}|, \bar{E}=| \Omega_{\mathcal{A}}\right\rangle\left\langle\Omega_{\mathcal{U}}\right|$. With an eye on (32), we now compute

$$
\begin{aligned}
\bar{E} a E & =S^{2}\left(e_{i}\right) f^{i} a E \\
& =f_{(1)}^{i} a_{(1)}\left\langle S^{2}\left(e_{i}\right), f_{(2)}^{i} a_{(2)}\right\rangle E \\
& =f^{n}\left\langle e_{n}, f_{(1)}^{i} a_{(1)}\right\rangle\left\langle S^{2}\left(e_{i}\right), f_{(2)}^{i} a_{(2)}\right\rangle E \\
& =f^{n}\left\langle e_{n} S^{2}\left(e_{i}\right), f^{i} a\right\rangle E \\
& =\langle a\rangle^{R} \delta_{\mathcal{A}}^{L} E .
\end{aligned}
$$

This simplifies further when $\delta_{\mathcal{A}}^{L}=\delta_{\mathcal{A}}^{R} \equiv \delta_{\mathcal{A}}$ - we then get

$$
\bar{E} a E=\langle a\rangle \delta_{\mathcal{A}} .
$$

\subsection{Fourier Transforms}

We work again with a general (i.e. not necessarily unimodular) FDHA. We define a right Fourier transform $\wedge^{R}: \mathcal{A} \rightarrow \mathcal{U}$ in terms of a right integral as follows

$$
\widehat{a}^{R} \equiv\left\langle a S^{-1}\left(f^{i}\right)\right\rangle^{R} e_{i}
$$

so that $(b$ in $\mathcal{A})$

$$
\left\langle\widehat{a}^{R}, b\right\rangle=\left\langle a S^{-1}(b)\right\rangle^{R} .
$$

We show now that the right Fourier transform is invertible

$$
\begin{aligned}
\left\langle e_{j} \widehat{a}^{R}\right\rangle^{R} f^{j} & =\left\langle a S^{-1}\left(f^{i}\right)\right\rangle^{R}\left\langle e_{j} e_{i}\right\rangle^{R} f^{j} \\
& =\left\langle a S^{-1}\left(f_{(2)}^{i}\right)\right\rangle^{R}\left\langle e_{i}\right\rangle^{R} f_{(1)}^{i} \\
& =\left\langle a_{(1)} S^{-1}\left(f_{(3)}^{i}\right)\right\rangle^{R}\left\langle e_{i}\right\rangle^{R} a_{(2)} S^{-1}\left(f_{(2)}^{i}\right) f_{(1)}^{i} \\
& =\left\langle a_{(1)} S^{-1}\left(f^{i}\right)\right\rangle^{R}\left\langle e_{i}\right\rangle^{R} a_{(2)} \\
& =\left\langle a_{(1)} S^{-1}\left(\delta_{\mathcal{A}}^{R}\right)\right\rangle^{R} a_{(2)} \\
& =\left\langle S^{-1}\left(\delta_{\mathcal{A}}^{R}\right)\right\rangle^{R} a \\
& =a .
\end{aligned}
$$

In the language of the previous section, when $\sigma^{\prime} \neq 0$, the Fourier transform allows the switching between $\left|\Omega_{\mathcal{U}}\right\rangle$ and $\left|\Omega_{\mathcal{A}}\right\rangle$. Indeed, we find

$$
\begin{aligned}
a E & =a S^{-1}\left(f^{i}\right) e_{i} \\
& =e_{i_{(2)}}\left\langle e_{i_{(1)}}, S^{-1}\left(a_{(2)} S^{-1}\left(f_{(1)}^{i}\right)\right)\right\rangle a_{(1)} S^{-1}\left(f_{(2)}^{i}\right) \\
& =e_{i_{(2)}}\left\langle S^{-2}\left(e_{i_{(1)}}\right), f_{(1)}^{i} S\left(a_{(2)}\right)\right\rangle a_{(1)} S^{-1}\left(f_{(2)}^{i}\right) \\
& =e_{j}\left\langle S^{-2}\left(e_{i}\right), f_{(1)}^{i} f_{(1)}^{j} S\left(a_{(2)}\right)\right\rangle a_{(1)} S^{-1}\left(f_{(2)}^{j}\right) S^{-1}\left(f_{(2)}^{i}\right) \\
& =e_{j}\left\langle S^{-2}\left(e_{i}\right) S^{-2}\left(e_{k}\right), f^{i} f_{(1)}^{j} S\left(a_{(2)}\right)\right\rangle a_{(1)} S^{-1}\left(f_{(2)}^{j}\right) S^{-1}\left(f^{k}\right) \\
& =e_{j}\left\langle S^{-2}\left(e_{k}\right)\right\rangle\left\langle f_{(1)}^{j} S\left(a_{(2)}\right)\right\rangle a_{(1)} S^{-1}\left(f_{(2)}^{j}\right) S^{-1}\left(f^{k}\right) \\
& =e_{j}\left\langle a_{(2)} S^{-1}\left(f_{(1)}^{j}\right)\right\rangle a_{(1)} S^{-1}\left(f_{(2)}^{j}\right)\left\langle S^{-3}\left(e_{k}\right)\right\rangle f^{k} \\
& =e_{j}\left\langle a S^{-1}\left(f^{j}\right)\right\rangle\left\langle e_{k}\right\rangle f^{k} \\
& =\hat{a} \delta_{\mathcal{A}},
\end{aligned}
$$


which, in terms of the action on right vacua, corresponds to

$$
a\left|\Omega_{\mathcal{U}}\right\rangle=\hat{a}\left|\Omega_{\mathcal{A}}\right\rangle
$$

One can easily check that ( $a$ in $\mathcal{A}, x$ in $\mathcal{U}$ )

$$
\widehat{x \triangleright a^{R}}=x \widehat{a}^{R} .
$$

Another familiar, in the unimodular case, property that survives when $\left\langle 1_{\mathcal{A}}\right\rangle=0$, is

$$
\widehat{f \star^{R}} g^{R}=\widehat{f}^{R} \widehat{g}^{R}
$$

where the right convolution $\star^{R}$ of $f, g$ in $\mathcal{A}$ is given by

$$
f \star^{R} g=g_{(1)}\left\langle f S^{-1}\left(g_{(2)}\right)\right\rangle^{R}
$$

( (46), together with the invertibility of $\hat{\bullet}^{R}$, guarantee the associativity of $\star^{R}$ ). On the other hand, the following property that is easily seen to hold in the unimodular case,

$$
\hat{\hat{a}}=S(a)
$$

does not hold, in general, for $\hat{\bullet}^{R}$ when $\left\langle 1_{\mathcal{A}}\right\rangle=0$.

\subsection{Further properties}

We give now the proof of a number of interesting formulas, valid for unimodular FDHAs. First, notice that

$$
\begin{aligned}
\widehat{S^{2}(a)} & =e_{i}\left\langle S^{2}(a) S^{-1}\left(f^{i}\right)\right\rangle \\
& =e_{i}\left\langle a S^{-3}\left(f^{i}\right)\right\rangle \\
& =S^{-2}\left(e_{i}\right)\left\langle a S^{-1}\left(f^{i}\right)\right\rangle \\
& =S^{-2}(\hat{a})
\end{aligned}
$$

where, in the second line, we used (19). Two useful lemmas follow

Lemma 4 For $\mathcal{A}$ a FDHA and $\sigma^{\prime} \neq 0$, it holds

$$
S^{-2}(a)=\left\langle a^{(\overline{1})}, S^{-2}\left(a^{(2)}(2)\right)\right\rangle a^{(2)}(1)
$$

for all a in $\mathcal{A}$.

Proof: We have (the notation is introduced in section 2)

$$
\begin{aligned}
a^{(\overline{1})} \otimes a^{(2)}{ }_{(1)} \otimes a^{(2)}{ }_{(2)} & =(\mathrm{id} \otimes \Delta)\left(S\left(e_{i}\right) e_{j} \otimes f^{i} a f^{j}\right) \\
& =S\left(e_{k}\right) S\left(e_{i}\right) e_{j} e_{l} \otimes f^{i} a_{(1)} f^{j} \otimes f^{k} a_{(2)} f^{l}
\end{aligned}
$$


which gives

$$
\begin{aligned}
\left\langle a^{(\overline{1})}, S^{-2}\left(a^{(2)}{ }_{(2)}\right)\right\rangle a^{(2)}{ }_{(1)} & =\left\langle S\left(e_{k}\right) S\left(e_{i}\right) e_{j} e_{l}, S^{-2}\left(f^{k}\right) S^{-2}\left(a_{(2)}\right) S^{-2}\left(f^{l}\right)\right\rangle f^{i} a_{(1)} f^{j} \\
& =\left\langle S\left(e_{k}\right) S\left(e_{i}\right) e_{j}\right\rangle\left\langle S^{-2}\left(f^{k}\right) S^{-2}\left(a_{(2)}\right)\right\rangle f^{i} a_{(1)} f^{j} \\
& =\left\langle S\left(e_{i}\right) e_{j}\right\rangle\left\langle S^{-2}\left(f_{(2)}^{i}\right) S^{-2}\left(a_{(2)}\right)\right\rangle f_{(1)}^{i} a_{(1)} f^{j} \\
& =\left\langle S\left(e_{i}\right) e_{j}\right\rangle\left\langle f^{i} a\right\rangle f^{j} \\
& =\left\langle S\left(e_{i}\right) e_{j}\right\rangle\left\langle S^{-1}(a) S^{-1}\left(f^{i}\right)\right\rangle f^{j} \\
& =\left\langle S\left(S^{-1}(a)\right) e_{j}\right\rangle f^{j} \\
& =\left\langle e_{j} S^{2}\left(S^{-1}(a)\right)\right\rangle S\left(f^{j}\right) \\
& =\left\langle e_{j} S^{-3}(a)\right\rangle S\left(f^{j}\right) \\
& =S^{-2}(a)
\end{aligned}
$$

where, in the last line, we used the formula for the inverse Fourier transform, eqn (42).

Lemma 5 For $\mathcal{A}$ a FDHA, it holds

$$
a^{(2)} S^{-1}\left(a^{(\overline{1})}\right) b=b a^{(2)} S^{-1}\left(a^{(\overline{1})}\right)
$$

for all $a, b$ in $\mathcal{A}$.

Proof: We have

$$
\begin{aligned}
a^{(2)} S^{-1}\left(a^{(\overline{1})}\right) b & =a^{(2)} b_{(1)}\left\langle S^{-1}\left(a^{(\overline{1})}{ }_{(2)}\right), b_{(2)}\right\rangle S^{-1}\left(a^{(\overline{1})}{ }_{(1)}\right) \\
& =b_{(1)}\left\langle a^{(2)(\overline{1})}, b_{(2)}\right\rangle a^{(2)(2)}\left\langle S^{-1}\left(a^{(\overline{1})}{ }_{(2)}\right), b_{(3)}\right\rangle S^{-1}\left(a^{(\overline{1})}{ }_{(1)}\right) \\
& =b_{(1)}\left\langle a^{(2)(\overline{1})} S^{-1}\left(a^{(\overline{1})}{ }_{(2)}\right), b_{(2)}\right\rangle a^{(2)(2)} S^{-1}\left(a^{(\overline{1})}{ }_{(1)}\right) \\
& =b_{(1)}\left\langle a^{(\overline{1})}{ }_{(3)} S^{-1}\left(a^{(\overline{1})}{ }_{(2)}\right), b_{(2)}\right\rangle a^{(2)} S^{-1}\left(a^{(\overline{1})}{ }_{(1)}\right) \\
& =b a^{(2)} S^{-1}\left(a^{(\overline{1})}\right)
\end{aligned}
$$

where, in the first and second line, we used the first and second of (4) respectively. At this point, we have enough machinery at our disposal to prove the following

Proposition 1 For $\mathcal{A}$ a FDHA and $\sigma^{\prime} \neq 0$, it holds

$$
\langle b a\rangle=\left\langle S^{-2}(a) b\right\rangle
$$

for all $a, b$ in $\mathcal{A}$.

Proof: We have

$$
\begin{aligned}
\bar{E} b a E & =\bar{E} b a^{(2)} \epsilon\left(S^{-1}\left(a^{(\overline{1})}\right)\right) E \\
& =\bar{E} b a^{(2)} S^{-1}\left(a^{(\overline{1})}\right) E \\
& =\bar{E} a^{(2)} S^{-1}\left(a^{(\overline{1})}\right) b E \\
& =\bar{E} S^{-1}\left(a^{(\overline{1})}{ }_{(1)}\right)\left\langle S^{-1}\left(a^{(\overline{1})}{ }_{(2)}\right), S^{-1}\left(a^{(2)}(2)\right)\right\rangle a^{(2)}{ }_{(1)} b E \\
& =\bar{E}\left\langle S^{-1}\left(a^{(\overline{1})}\right), S^{-1}\left(a^{(2)}{ }_{(2)}\right)\right\rangle a^{(2)}{ }_{(1)} b E \\
& =\bar{E} S^{-2}(a) b E
\end{aligned}
$$


where in the third line we used (52) and in the last one, (50). The proposition follows now from (39).

It is interesting to compare (54) with the formula one can derive in the presence of a universal $R$-matrix $\mathcal{R}$ in $\mathcal{U}$. The result in this case is contained in

Proposition 2 Let $\mathcal{A}$ be a dual quasitriangular Hopf algebra and $\langle\cdot\rangle$ a biinvariant integral on it. It holds

$$
\langle b a\rangle=\left\langle S^{2}(a \triangleleft s) b\right\rangle .
$$

for all $a, b$ in $\mathcal{A}$, where

$$
s=c u^{-2}, \quad u=S\left(\mathcal{R}^{(2)}\right) \mathcal{R}^{(1)}, \quad c=u S(u)
$$

$\left(s, u, c\right.$ in $\left.\mathcal{U} \sim \mathcal{A}^{*}\right)$ and $a \triangleleft s \equiv\left\langle s_{(1)}, a\right\rangle s_{(2)}$.

Proof: The commutation relations in $\mathcal{A}$ can be written in the form

$$
b a=\left\langle\mathcal{R}, a_{(1)} \otimes b_{(1)}\right\rangle a_{(2)} b_{(2)}\left\langle\mathcal{R}^{-1}, a_{(3)} \otimes b_{(3)}\right\rangle
$$

(this is the dual version of (11)). It can also be shown that the element $u$ defined above implements the square of the antipode in $\mathcal{U}$ acccording to

$$
S^{2}(x)=u x u^{-1}
$$

for all $x$ in $\mathcal{U}[7]$ - its inverse is given by $u^{-1}=\mathcal{R}^{(2)} S^{2}\left(\mathcal{R}^{(1)}\right)$. We can then write

$$
\begin{aligned}
\langle b a\rangle & =\left\langle\mathcal{R}, a_{(1)} \otimes b_{(1)}\right\rangle\left\langle a_{(2)} b_{(2)}\right\rangle\left\langle\mathcal{R}^{-1}, a_{(3)} \otimes b_{(3)}\right\rangle \\
& =\left\langle\mathcal{R}, a_{(1)} \otimes b_{(1)} S\left(b_{(2)}\right) S\left(a_{(2)}\right)\right\rangle\left\langle a_{(3)} b_{(3)}\right\rangle\left\langle\mathcal{R}^{-1}, a_{(5)} \otimes b_{(5)} S^{-1}\left(b_{(4)}\right) S^{-1}\left(a_{(4)}\right)\right\rangle \\
& =\left\langle\mathcal{R}, a_{(1)} \otimes S\left(a_{(2)}\right)\right\rangle\left\langle a_{(3)} b\right\rangle\left\langle\mathcal{R}^{-1}, a_{(5)} \otimes S^{-1}\left(a_{(4)}\right)\right\rangle \\
& =\left\langle S(u), a_{(1)}\right\rangle\left\langle a_{(2)} b\right\rangle\left\langle u^{-1}, a_{(3)}\right\rangle .
\end{aligned}
$$

However,

$$
\begin{aligned}
S(u) x u^{-1} & =S(u) u^{-1} u x u^{-1} \\
& =s S^{2}(x) .
\end{aligned}
$$

Then, for arbitrary $x$ in $\mathcal{U}$, we have

$$
\begin{aligned}
\left\langle S(u), a_{(1)}\right\rangle\left\langle x, a_{(2)}\right\rangle\left\langle u^{-1}, a_{(3)}\right\rangle & =\left\langle S(u) x u^{-1}, a\right\rangle \\
& =\left\langle s S^{2}(x), a\right\rangle \\
& =\left\langle x, S^{2}(a \triangleleft s)\right\rangle
\end{aligned}
$$

therefore

$$
\left\langle S(u), a_{(1)}\right\rangle a_{(2)}\left\langle u^{-1}, a_{(3)}\right\rangle=S^{2}(a \triangleleft s) ;
$$

substituting in (59) we get (55).

Comparison with (54) and use of (27) leads to the relation $S^{4}(a)=a \triangleleft s^{-1}$ which in the dual implies $S^{4}(x)=s^{-1} x$ and therefore (by taking $x=1_{\mathcal{U}}$ )

$$
S^{4}(x)=x
$$


for all $x$ in $\mathcal{U}$ (a different proof of this has been given in [20]). Equations (54) and (61) have been proven above only in the finite dimensional case (the latter assuming quasitriangularity as well). On the other hand, (55) and the following versions of it which are proved similarly, hold for all quasitriangular Hopf algebras with biinvariant integral

$$
\begin{aligned}
\langle b a\rangle & =\left\langle a S^{2}\left(s^{-1} \triangleright b\right)\right\rangle \\
& =\left\langle S^{-2}(s \triangleright a) b\right\rangle \\
& =\left\langle\left(u^{-1} \triangleright b\right)(a \triangleleft u)\right\rangle .
\end{aligned}
$$

We close this section with the remark that $\langle b a\rangle=\left\langle S^{2}(a) b\right\rangle$ has been shown to hold for unimodular, finite dimensional ribbon Hopf algebras (see [19]). Using (61) in (54) we conclude that it actually holds for (the wider class of) quasitriangular FDHAs.

\section{Integration On Braided Hopf Algebras}

We transcribe here the main results of section 3 to the case of FDBHAs, using the Ndimensional quantum superplane as a concrete example.

\subsection{Preliminaries}

\subsubsection{The quantum superplane}

Let us review briefly the basics of the construction of the quantum superplane [25, 27]. As is typical in the study of quantum spaces, one deals with the associative, noncommutative algebra $\mathcal{X}$ generated by $1_{\mathcal{X}}$ and the coordinate functions $\xi_{i}, i=1, \ldots, N$ on the quantum superplane satisfying the commutation relations

$$
\xi_{2} \xi_{1}=-q \hat{R}_{12} \xi_{2} \xi_{1}
$$

(we work with the " $q^{-1}$ " version [4] - we remind the reader that $\hat{R}_{12}\left(q^{-1}\right)=\hat{R}_{21}^{-1}(q)$ ). The derivatives $\sigma_{i}, i=1, \ldots, N$, dual to the above coordinates, generate (together with $1_{\mathcal{D}}$ ) the algebra $\mathcal{D}$ with commutation relations

$$
\sigma_{1} \sigma_{2}=-q \sigma_{1} \sigma_{2} \hat{R}_{12}
$$

The coordinate-derivative duality is encoded in the cross relations

$$
\sigma_{i} \xi_{j}=\delta_{i j}-q \hat{R}_{m j, n i}^{-1} \xi_{n} \sigma_{m}
$$

We denote the combined coordinate - derivative algebra by $\mathcal{P}$. In analogy with the treatment of the quantum plane in [3], one can enlarge $\mathcal{P}$ by the introduction of displacements $\eta_{i}, \tau_{i}, i=$ $1, \ldots, N$ for the coordinates and derivatives respectively, satisfying

$$
\begin{gathered}
\eta_{2} \eta_{1}=-q \hat{R}_{12} \eta_{2} \eta_{1}, \quad \tau_{1} \tau_{2}=-q \tau_{1} \tau_{2} \hat{R}_{12}, \quad \tau_{i} \eta_{j}=\delta_{i j}-q \hat{R}_{m j, n i}^{-1} \eta_{n} \tau_{m} \\
\xi_{2} \eta_{1}=-q \hat{R}_{12}^{-1} \eta_{2} \xi_{1}, \quad \sigma_{i} \eta_{j}=-q^{-1} \hat{R}_{k j, l i} \eta_{l} \sigma_{k} \\
\xi_{i} \tau_{j}=-q^{-1} D_{l a} \hat{R}_{i a, b k} D_{b j}^{-1} \tau_{l} \xi_{k}, \quad \sigma_{1} \tau_{2}=-q \tau_{1} \sigma_{2} \hat{R}_{12}^{-1}
\end{gathered}
$$


As seen from above, the $\eta$ 's are taken to be just a second copy of the $\xi$ 's but are endowed with nontrivial statistics with both the $\xi$ 's and the $\sigma$ 's - analogous remarks hold for the $\tau$ 's. The remarkable property of (68) is that the displaced coordinates $\xi_{i}+\eta_{i}$ and derivatives $\sigma_{i}+\tau_{i}$ still satisfy (63) and (64) respectively while the entire enlarged algebra is covariant under the $G L_{q}(N)$-coaction

$$
\begin{aligned}
\xi_{i} \mapsto \xi_{i}^{\prime} & =\xi_{j} \otimes S\left(A_{i j}\right) \\
\eta_{i} \mapsto \eta_{i}^{\prime} & =\eta_{j} \otimes S\left(A_{i j}\right) \\
\sigma_{i} \mapsto \sigma_{i}^{\prime} & =\sigma_{j} \otimes S^{2}\left(A_{j i}\right) \\
\tau_{i} \mapsto \tau_{i}^{\prime} & =\tau_{j} \otimes S^{2}\left(A_{j i}\right) .
\end{aligned}
$$

We will often drop the tensor product sign in the following.

\subsubsection{Braiding}

Suppose $\mathcal{U}$ is a quasitriangular Hopf algebra (with universal $R$-matrix $\mathcal{R}$ ) that acts from the left on two algebras $V, W$. One can, in this case, form the braided tensor product $W \underline{\otimes} V$ in which $V, W$ are trivially embedded as subalgebras but have nontrivial statistics, given by $(v$ in $V, w$ in $W$ )

$$
\begin{aligned}
(\underline{1} \underline{\otimes} v)(w \underline{\otimes} 1) & \equiv \Psi(v \otimes w) \\
& =\tau \circ\left(\mathcal{R}^{(1)} \triangleright v \otimes \mathcal{R}^{(2)} \triangleright w\right) \\
& =w^{(1)} \underline{\otimes} v^{(1)}\left\langle\mathcal{R}, v^{\left(2^{\prime}\right)} \otimes w^{\left(2^{\prime}\right)}\right\rangle .
\end{aligned}
$$

We have expressed above the action of $\mathcal{U}$ on $V, W$ in terms of the dual coaction of $\mathcal{A} \sim \mathcal{U}^{*}$. The first line of (73) also defines the braided transposition $\Psi: V \otimes W \rightarrow W \otimes V$ for which it holds in general $\Psi^{2} \neq$ id (due to $\mathcal{R}^{\prime} \mathcal{R} \neq 1$ ). For a detailed discussion of the properties of $\Psi$ see e.g. [17].

\subsubsection{The quantum superplane as a braided Hopf algebra}

The concept of braided tensor products provides a natural framework for an elegant description of the quantum superplane as a braided Hopf algebra. We give here, for completeness, an outline of this approach - more details can be found in [17]. Essential to this description is the use of diagrams which encode neatly the braiding information. The maps $\Psi$ and $\Psi^{-1}$ are represented by the diagrams $\boldsymbol{X}$ and $\mathcal{Y}$ respectively. For the algebra $\mathcal{X}$, the map $\xi_{i} \mapsto \xi_{i} \underline{\otimes}+1 \underline{\otimes} \xi_{i} \equiv \eta_{i}+\xi_{i}$ is regarded as a braided coproduct $\underline{\Delta}: \mathcal{X} \rightarrow \mathcal{X} \underline{\otimes} \mathcal{X}$ (extended (braided) multiplicatively on the whole $\mathcal{X}$ ). Diagramaticaly this appears as
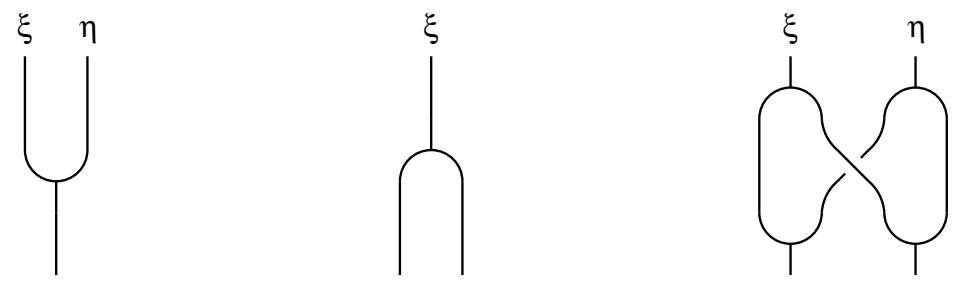

where the first two vertices denote the product and coproduct in $\mathcal{X}$ and the third diagram expresses the braided multiplicativity of $\triangleq(\xi, \eta$ etc. denote generic elements of $\mathcal{X})$. One also has a matching counit and antipode with $\epsilon, S, S^{2}, S^{-1}, S^{-2}$ denoted respectively by 
(†)

and satisfying braided versions of the familiar Hopf algebra identities, e.g.

$$
\Theta=1=
$$

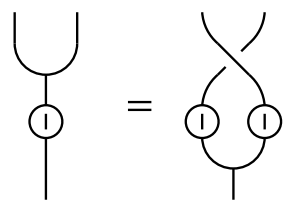

A particularly important requirement on the braiding, which $\Psi$ of (73) satisfies, is that one should be able to move crossings past all vertices and boxes, e.g.
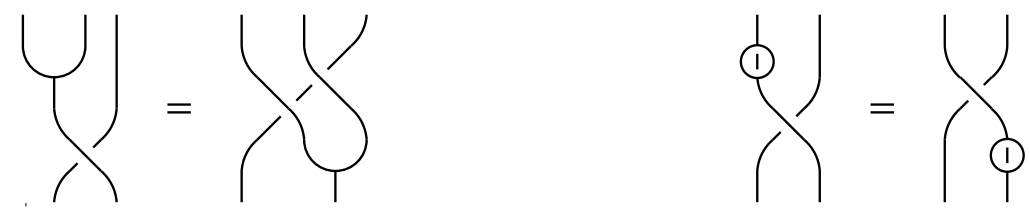

should hold. Exactly analogous treatment is possible for $\mathcal{D}$, the algebra of derivatives. The braided coproduct is given by $\underline{\Delta}\left(\sigma_{i}\right)=\sigma_{i} \underline{\otimes} 1+\underline{\underline{\otimes}} \sigma_{i} \equiv \tau_{i}+\sigma_{i}$ and the corresponding diagrams are an exact copy of those for $\mathcal{X}$ (we will use in them the letters $\sigma$, $\tau$ etc. to denote generic elements of $\mathcal{D}$ ). One can now combine $\mathcal{X}$ and $\mathcal{D}$ to form a braided semidirect product $\mathcal{X} \rtimes \mathcal{D}$. We need for this a braided action of $\mathcal{D}$ on $\mathcal{X}$ which is given by the second of the following diagrams
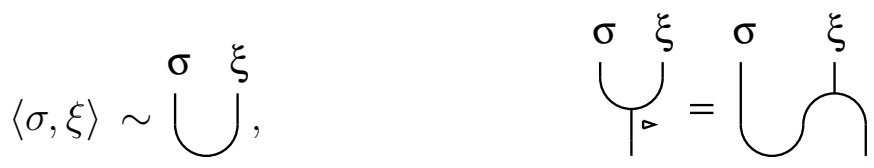

while the first one simply depicts the pairing between a derivative and a function (defined as the counit of the derivative of the function). When viewed upside - down, the first diagram stands for the canonical element $\phi^{i} \underline{\otimes} \epsilon_{i} \in \mathcal{X} \underline{\otimes \mathcal{D}}$ (notice the reversal of order in the tensor product) with $\left\langle\epsilon_{i}, \phi^{j}\right\rangle=\delta_{i}^{j}$. Both the inner product and the canonical element are assumed invariant under $\Delta_{\mathcal{A}}$ :

$$
\begin{aligned}
\left\langle p^{(1)}, x^{(1)}\right\rangle p^{\left(2^{\prime}\right)} x^{\left(2^{\prime}\right)} & =\langle p, x\rangle 1_{\mathcal{A}} \\
\phi^{i(1)} \underline{\otimes} \epsilon_{i}^{(1)} \otimes \phi^{i\left(2^{\prime}\right)} \epsilon_{i}{ }^{\left(2^{\prime}\right)} & =\phi^{i} \underline{\otimes} \epsilon_{i} \otimes 1_{\mathcal{A}} .
\end{aligned}
$$

The product - coproduct duality between $\mathcal{X}$ and $\mathcal{D}$ is taken to be
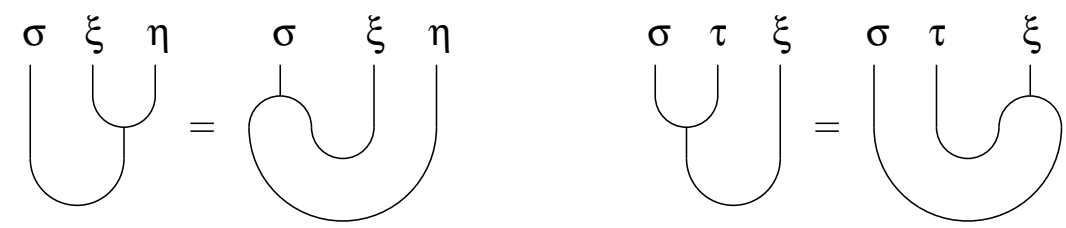

Notice that this differs from the standard convention in the unbraided case. As a result, to get the unbraided version of any diagrammatic equation that appears in the following, one should translate the diagrams, ignoring the braiding information, into the language of section 2 and then set $\Delta \rightarrow \Delta^{\prime}, S \rightarrow S^{-1}$. Again, viewing the diagrams upside - down reveals additional 
(dual) information - in the case of the diagrams above, one discovers two basic properties of the canonical element (compare with the first two of (6i)).

The commutation relations in the semidirect product (i.e. the braided analogue of (四)) are
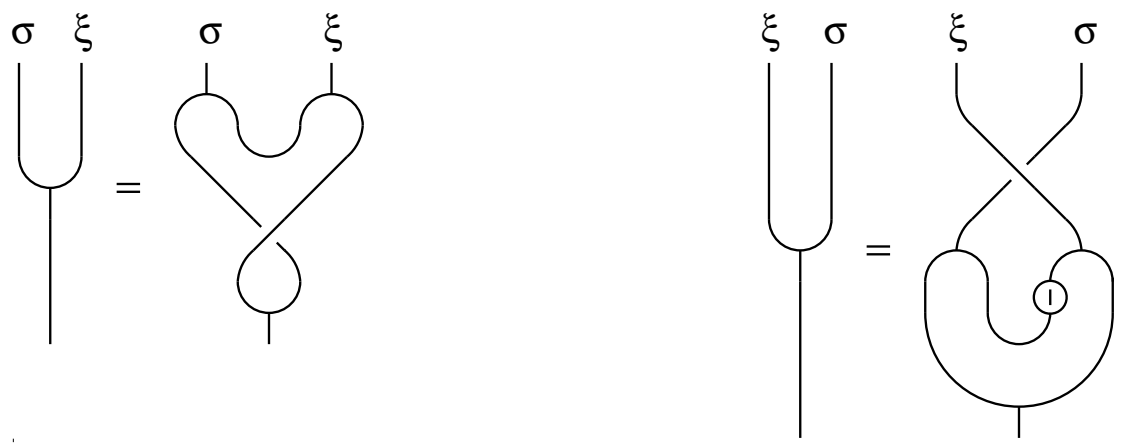

We close this review with a technical remark. If one computes the $\xi-\xi$ braiding given by (73) (with $V=W=\mathcal{X}$ ), using the coaction (69), one fails to reproduce the $\xi-\eta$ commutation relations of (67) - the result is off by a $q$ factor (similarly for the rest of (67), (68)). To remedy this, one can enlarge $\mathcal{A}$ by a grouplike central element $g$ (the dilaton, see [16]), the inner product of which with $\mathcal{R}$ is given by

$$
\left\langle\mathcal{R}, g^{a} \otimes g^{b}\right\rangle=(-q)^{-a b}, \quad\left\langle\mathcal{R}, g^{a} \otimes A_{i j}\right\rangle=\left\langle\mathcal{R}, A_{i j} \otimes g^{a}\right\rangle=\delta_{i j} .
$$

We will call the enlarged algebra $\tilde{\mathcal{A}}$. Setting $A \rightarrow g A$ in the rhs of (69)- (72) gives an $\tilde{\mathcal{A}}$-coaction on the quantum superplane which reproduces, via (73), the commutation relations (67), (68).

\subsection{The invariant integral}

\subsubsection{First definition and problems}

The integral we are looking for is a linear map $\langle\cdot\rangle: \mathcal{X} \rightarrow C$ which is translationally invariant in the following sense [25]

$$
\left\langle\sigma_{i} f(\xi)\right\rangle=0, \quad i=1, \ldots, N \quad \forall f \in \mathcal{X} .
$$

An equivalent formulation of invariance is [3]

$$
\langle f(\xi+\eta)\rangle=1_{\mathcal{X}}\langle f(\xi)\rangle \quad \forall f \in \mathcal{X}
$$

or, in braided Hopf algebra language,

$$
f_{\underline{(1)}}\left\langle\underline{f_{(2)}}\right\rangle=1_{\mathcal{X}}\langle f\rangle \quad \forall f \in \mathcal{X} .
$$

Representing the integral with a rhombus, we want it to satisfy

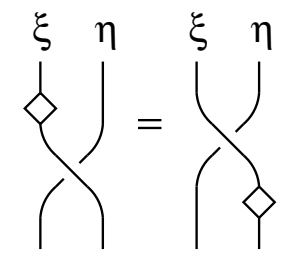


a requirement which, as one can easily see, cannot, in general, be satisfied. Indeed, we only need consider the classical fermionic line with coordinate $\xi$ and displacement $\eta$ satisfying $\xi^{2}=\eta^{2}=0, \xi \eta=-\eta \xi$. Taking $\xi, \eta$ to stand for themselves in the diagram above, we find that the lhs is $\eta\langle\xi\rangle$ (since $\langle\xi\rangle$, being a number, braids trivially), while the rhs is $\eta\langle-\xi\rangle=$ $-\eta\langle\xi\rangle$, implying $\langle\xi\rangle=0$ which contradicts the known Berezin result. We conclude that $\langle f(\xi)\rangle$, assumed invariant (and nontrivial), cannot both be a number and satisfy the property expressed in the diagram above.

\subsubsection{An improved definition}

Our treatment, in section 3.2, of the integral on FDHAs points to a simple solution to the above problem. We recall that there, the quantity that naturally emerged, in our algebraic formulation in terms of the modified trace formula, was the numerical integral $\langle\cdot\rangle$ times a delta function (as in the lhs of (22)). Motivated by this, we define a new integral $\langle\langle\cdot\rangle\rangle^{L}: \mathcal{X} \rightarrow k \delta_{\mathcal{X}}^{R}$ (with $k$, in our case, the complex numbers) as follows

$$
\langle\langle\xi\rangle\rangle^{L}=\langle\xi\rangle^{L} \delta_{\mathcal{X}}^{R}
$$

The output braid of the integration rhombus in our diagrams will stand accordingly for a numerical multiple of $\delta_{\mathcal{X}}^{R}$. The rhombus' inner workings are exposed in the diagram below

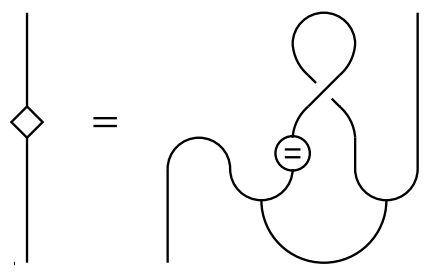

To get the product $\langle\sigma\rangle^{R}\langle\xi\rangle^{L}$ one should pair the output braid with $\sigma$. Whether (87), in its present or a suitably modified form, applies to the infinite dimensional case (and under what conditions), is a direction for future work (one can easily see that, in certain such cases, the rhs of (87) diverges).

Lemma 6 The integral $\langle\langle\cdot\rangle\rangle^{L}$ defined by (87) is nontrivial for every FDBHA.

Proof: Using $\left(S^{-1} \otimes \mathrm{id}\right) \circ \Psi^{-1}\left(\phi^{i} \otimes \epsilon_{i}\right)$ as input to the braided version of $\Theta$ (shown below), we find

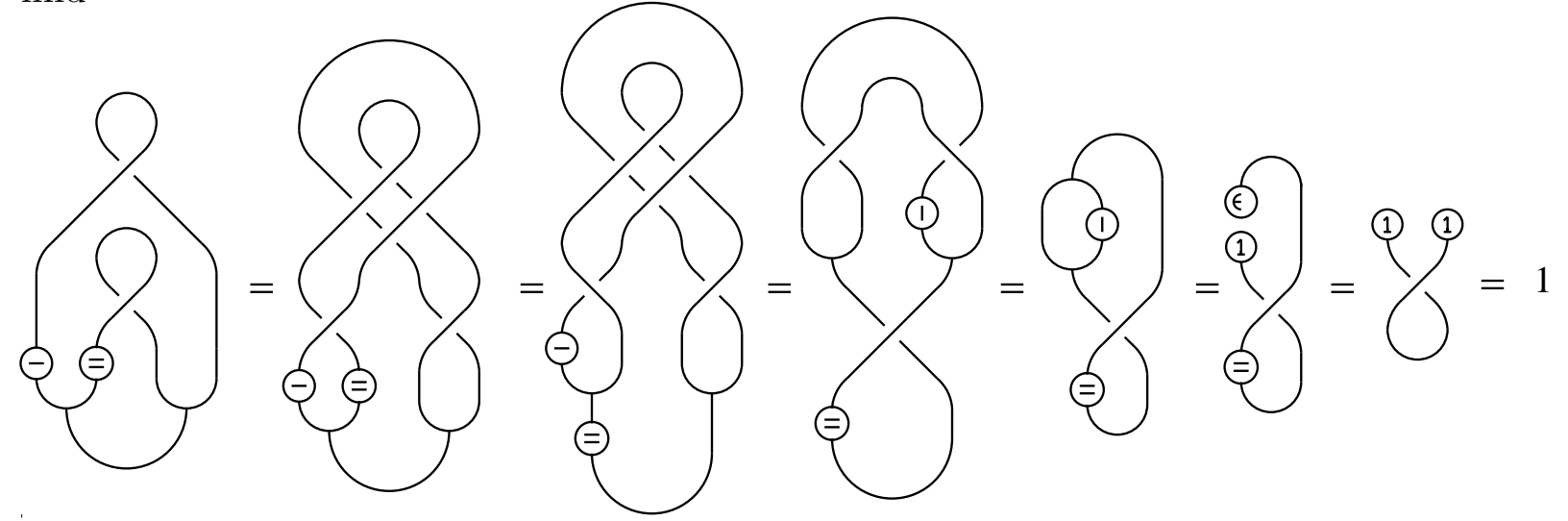

from which nontriviality follows. 


\subsubsection{Braided vacuum projectors}

We denote in the following by $\mathcal{E}, \overline{\mathcal{E}}$ the braided analogues of $E, \bar{E}$. They are given by

$$
\mathcal{E}=\bigcap_{1}, \quad \overline{\mathcal{E}}=\bigodot_{1}
$$

The proof of the (analogue of) (34) is as follows

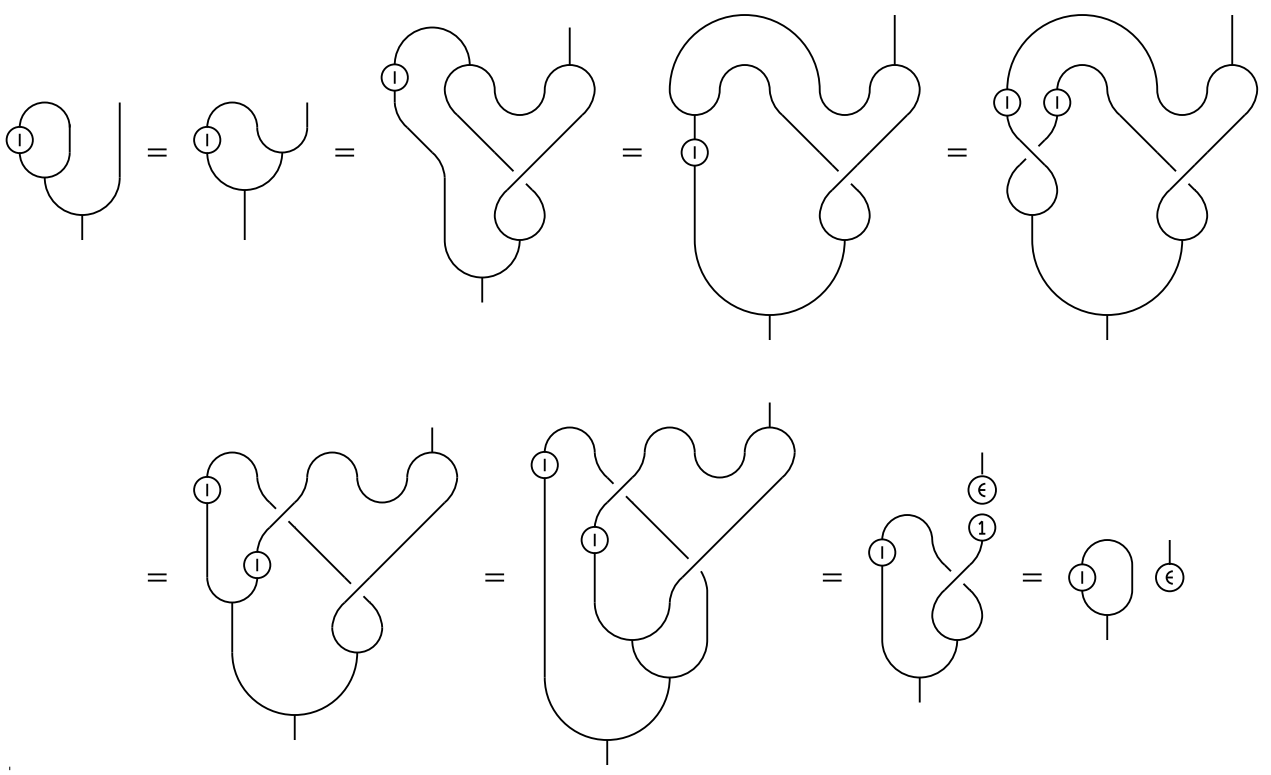

As before, $\overline{\mathcal{E}} a \mathcal{E}$ is a multiple of $\delta_{\mathcal{X}}^{R} \mathcal{E}$ - when $\delta_{\mathcal{X}}^{R}=\delta_{\mathcal{X}}^{L} \equiv \delta_{\mathcal{X}}$, it becomes a multiple of $\delta_{\mathcal{X}}$. Notice that, with $\delta_{\mathcal{X}}^{R}$ defined via $\left\langle\tau, \delta_{\mathcal{X}}^{R}\right\rangle=\langle\tau\rangle^{R}$, one gets $\xi \delta_{\mathcal{X}}^{R}=\epsilon(\xi) \delta_{\mathcal{X}}^{R}$ - the difference in the order of multiplication, compared to (14), is due to the second of (79).

The explicit computation of $\overline{\mathcal{E}}$ is simplified by the following identity

$$
\Psi^{-1}\left(\phi^{i} \otimes \epsilon_{i}\right)=S\left(u^{-1}\right) \triangleright \epsilon_{i} \otimes \phi^{i}
$$

which is easily proved using the invariance of the canonical element.

Inspection of our definition reveals the braided version of the trace formula (20) for the numerical integral $\langle\xi\rangle_{\underline{t r}}^{R}$ - it is given by

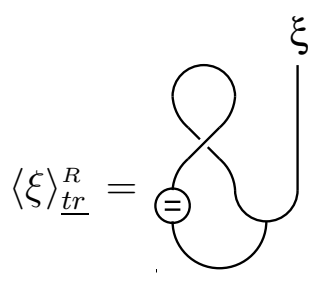

The proof of invariance is as follows 


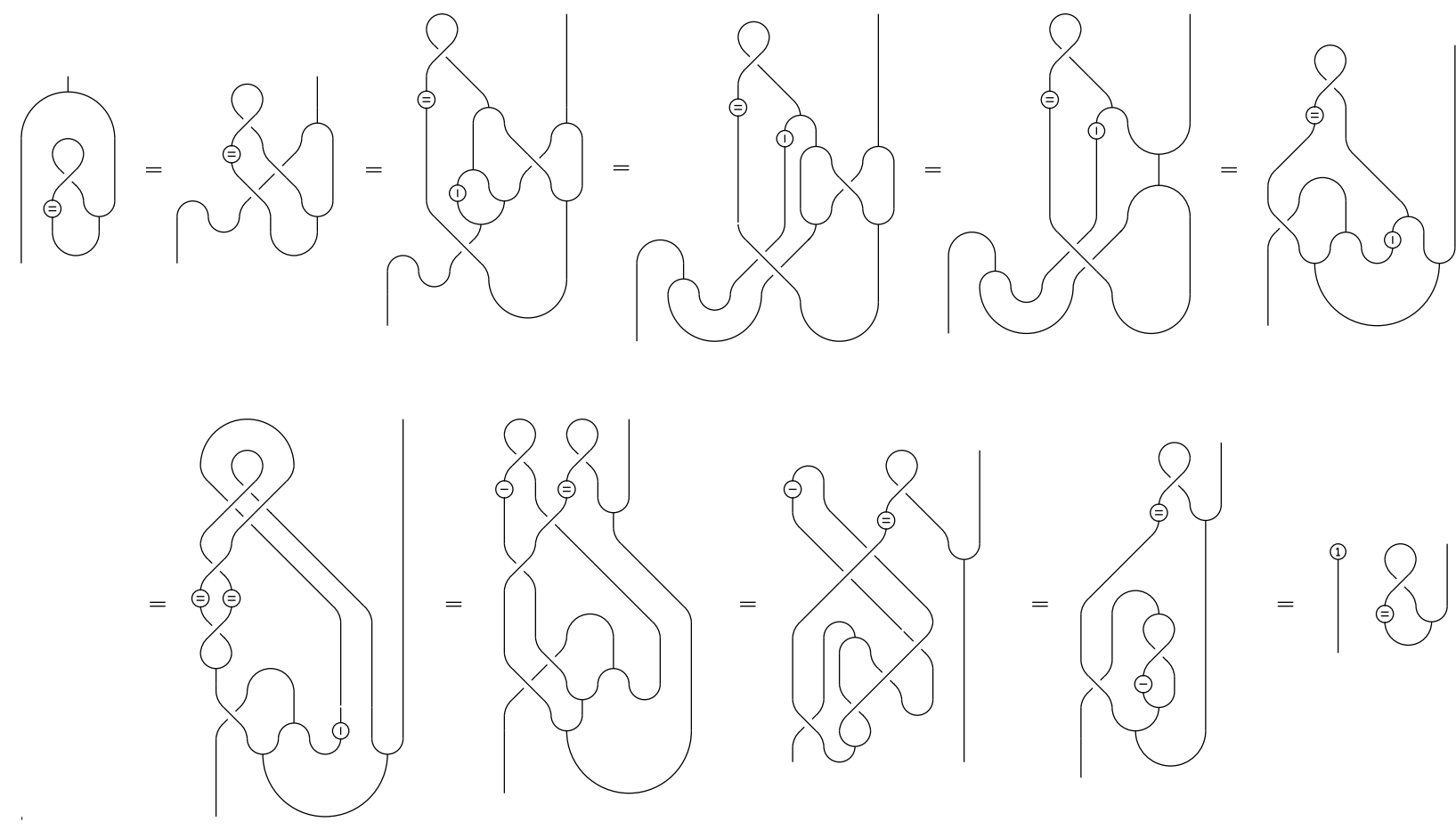

To find out under what conditions it becomes trivial, we have to derive the braided version of (29). Ommiting the somewhat lengthy diagrammatic proof, which parallels that of Lemma 3, we state

Lemma 7 For $\mathcal{X}, \mathcal{D}$ dually paired FDBHAs, it holds
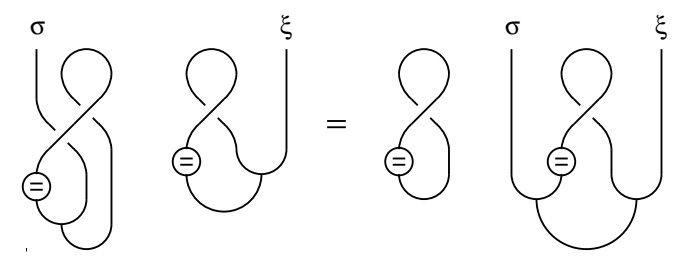

In analogy with the unbraided case, when $\underline{\sigma} \equiv\left\langle 1_{\mathcal{X}}\right\rangle_{\underline{t r}}^{R}=0,\langle\cdot\rangle_{\underline{t r}}^{R}$ is trivial; when $\underline{\sigma} \neq 0,\langle\cdot\rangle_{\underline{t r}}^{R}$ provides a nontrivial integral in $\mathcal{X}$. For the existence of integrals on FDBHAs and properties of them, see also [13, 14]. An analogous definition for the numerical braided integral (and a different proof of its invariance) can be found in [18.

\subsubsection{Braided Fourier transforms}

Transcribing (40), we define the Fourier transform $\hat{f}$ of the element $\xi$ of a FDBHA $\mathcal{X}$ by the equation

$$
\hat{\xi} \equiv\left\langle\xi S\left(\phi^{i}\right)\right\rangle \epsilon_{i}
$$

or, in pictures,

$$
\stackrel{\xi}{\xi}=\underbrace{\xi}_{\}}
$$


(this differs from earlier definitions [10] by the use of the nonbosonic integral $\langle\langle\cdot\rangle\rangle$ ). The output braid on the right stands for what one usually calls the Fourier transform of $\xi$ (an element of $\mathcal{D}$, the dual of $\mathcal{X}$ ) while the one on the left stands for the delta function in $\mathcal{X}$ that is produced by the integration and which ensures the correct braiding behavior of $\hat{\imath}$. There is also a notion of braided convolution of functions, defined by

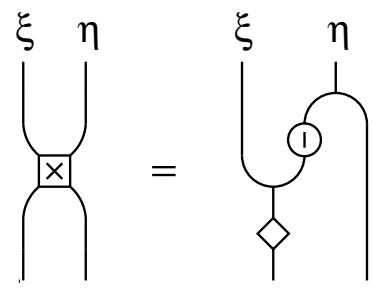

Again, the output braid on the left only carries a delta function in $\mathcal{X}$. The following basic properties can be shown to hold
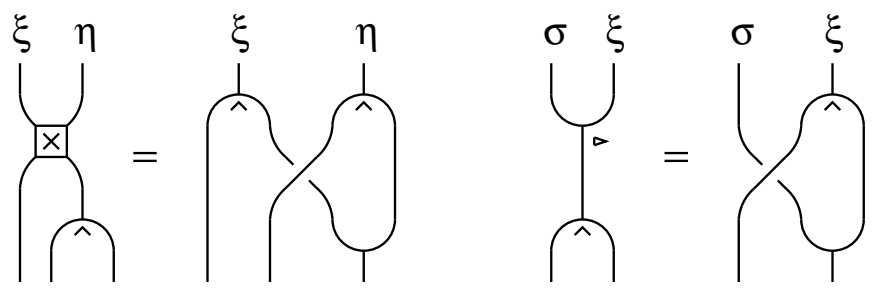

\subsection{Integration on the quantum superplane}

We apply now the general formalism developed above to the problem of integration on the quantum superplane. Our starting point will be the vacuum projector construction of section 4.2 .3 - notice that although $\left\langle 1_{\mathcal{A}}\right\rangle=0$ in this case, the integral is nevertheless biinvariant so we expect (39) to hold. For the canonical element we find

$$
\phi^{i} \underline{\otimes} \epsilon_{i}=e_{q^{-1}}\left(\xi_{i} \underline{\otimes} \sigma_{i}\right)
$$

where

$$
e_{q}(x)=\sum_{k=0}^{\infty} \frac{1}{[k]_{q} !} x^{k}, \quad[k]_{q}=\frac{1-q^{2 k}}{1-q^{2}}, \quad[k]_{q} !=[1]_{q}[2]_{q} \ldots[k]_{q}, \quad[0]_{q} \equiv 1
$$

(compare with the vacuum projectors for the quantum plane in [3]). The commutation relations (63), (64) imply $\xi_{i}^{2}=\sigma_{i}^{2}=0$ for $i=1, \ldots, N$ which gives $\left(\xi_{i} \underline{\otimes} \sigma_{i}\right)^{N+1}=0$. Using the braiding relations (67), the second of which can also be written as

$$
\Psi\left(\sigma_{i} \otimes \xi_{j}\right)=-q^{-1} \hat{R}_{k j, l i} \xi_{l} \otimes \sigma_{k},
$$

we can expand $\left(\xi_{i} \underline{\otimes} \sigma_{i}\right)^{k}$ in $\left.\underline{95}\right)$ to find

$$
\phi^{i} \underline{\otimes} \epsilon_{i}=\sum_{k=0}^{N} \frac{q^{-k(k-1)}}{[k]_{q^{-1}} !} \xi_{i_{1}} \ldots \xi_{i_{k}} \otimes \sigma_{i_{k}} \ldots \sigma_{i_{1}} .
$$

With the antipode being given by

$$
S\left(\xi_{i_{1}} \ldots \xi_{i_{k}}\right)=(-1)^{k} q^{k(k-1)} \xi_{i_{1}} \ldots \xi_{i_{k}}
$$


and using (89) we find

$$
\begin{aligned}
\mathcal{E} & =\sum_{k=0}^{N} \frac{(-1)^{k}}{[k]_{q^{-1}} !} \xi_{i_{1}} \ldots \xi_{i_{k}} \sigma_{i_{k}} \ldots \sigma_{i_{1}} \\
\overline{\mathcal{E}} & =\sum_{k=0}^{N} \frac{(-1)^{k} q^{k}}{[k]_{q} !} D_{i_{1} j_{1}} \ldots D_{i_{k} j_{k}} \sigma_{i_{k}} \ldots \sigma_{i_{1}} \xi_{j_{1}} \ldots \xi_{j_{k}} .
\end{aligned}
$$

It will be convenient in the following to express $\overline{\mathcal{E}}$ in the alternative form

$$
\overline{\mathcal{E}}=\sum_{k=0}^{N} \frac{(-1)^{k} q^{k(k-2 N+1)}}{[k]_{q} !}\left([N]_{q}-\xi \cdot \sigma\right)\left([N-1]_{q}-\xi \cdot \sigma\right) \ldots\left([N-k+1]_{q}-\xi \cdot \sigma\right)
$$

where $\xi \cdot \sigma \equiv \xi_{i} \sigma_{i}$ (this form makes the invariance under $\Delta_{\mathcal{A}}$ evident - a similar expression exists for $\mathcal{E})$. Using the commutation relation $\xi \cdot \sigma \xi_{j}=\xi_{j}\left(1+q^{2} \xi \cdot \sigma\right)$ and the fact that $\xi \cdot \sigma \mathcal{E}=0$, we can now compute the integral of an arbitrary monomial $\xi_{i_{1}} \ldots \xi_{i_{r}}(r<N)$

$$
\overline{\mathcal{E}} \xi_{i_{1}} \ldots \xi_{i_{r}} \mathcal{E}=\left(\sum_{k=0}^{A} \frac{(-1)^{k} q^{k(k-2 A+1)}[A]_{q} !}{[k]_{q} ![A-k]_{q} !}\right) \xi_{i_{1}} \ldots \xi_{i_{r}} \mathcal{E}
$$

where $A \equiv N-r$. For the sum in parentheses, one can set

$$
S(z)=\sum_{k=0}^{A} \frac{(-1)^{k} q^{k(k-2 A+1)}[A]_{q} !}{[k]_{q} ![A-k]_{q} !} z^{k}
$$

Introducing a Jackson derivative $\partial_{z}$, satisfying $\partial_{z} z=1+q^{2} z \partial_{z}$, we find from (104)

$$
\partial_{z} S(z)=\frac{1}{q^{-2}-1} S(z)-\frac{q^{-2 A}}{q^{-2}-1} S\left(q^{2} z\right)
$$

On the other hand, it holds

$$
\partial_{z} S(z)=\frac{S\left(q^{2} z\right)-S(z)}{q^{2}-1}
$$

comparison with (105) shows that

$$
\frac{S\left(q^{2} z\right)}{S(z)}=\frac{1-q^{2} z}{1-q^{-2(A-1)} z}
$$

from which we find

$$
S(z)=(1-z)\left(1-q^{-2} z\right) \ldots\left(1-q^{-2(A-1)} z\right),
$$

implying that $S(1)=0$ and therefore that

$$
\left\langle\xi_{i_{1}} \ldots \xi_{i_{r}}\right\rangle=0 \quad 0 \leq r<N
$$

For $r=N$, the integrand is (a multiple of) a delta function and its numerical integral is evidently (a multiple of) 1 . We conclude that the quantum Berezin integral in $N$ dimensions is essentially undeformed. As expected, $\langle\cdot\rangle_{\underline{t r}}^{R}$ is trivial in this case, as the reader can easily verify. 


\subsection{Remarks on the integral on the quantum plane}

We make here a few remarks about the transformation properties of the invariant integral on the quantum plane 25]. We denote by $x_{i}, i=1, \ldots, N$ the coordinate functions on it and by $\partial_{i}, i=1, \ldots, N$ the dual derivatives, satisfying

$$
x_{1} x_{2}=q^{-1} \hat{R}_{12} x_{1} x_{2}, \quad \partial_{2} \partial_{1}=q^{-1} \hat{R}_{12} \partial_{2} \partial_{1}, \quad \partial_{i} x_{j}=\delta_{i j}+q \hat{R}_{j l . i k} x_{k} \partial_{l} .
$$

The above algebra is covariant under the transformation $x \rightarrow \Delta_{\mathcal{A}}(x)=x A, \partial \rightarrow \Delta_{\mathcal{A}}(\partial)=$ $\partial S\left(A^{T}\right)$ with $A$ a $G L_{q}(N)$ matrix (we ommit the tensor product symbol). We assume an integral $\langle\langle\cdot\rangle\rangle$ exists, defined on a suitable class of functions, satisfying translational invariance (in the spirit of (82)) and braiding correctly, i.e. according to (85). In the classical case of integration on the $\mathrm{N}$ - dimensional plane, one finds the transformation property

$$
\int f(x A) d x=\frac{1}{\operatorname{det}(A)} \int f(x) d x
$$

where $A$ is a $G L(N)$ matrix. We now show that a similar property holds in the quantum case. We remark first that (85) implies

$$
\Delta_{\mathcal{A}}(\langle\langle f(x)\rangle\rangle)=\langle\langle f(x A)\rangle\rangle=\left\langle\left\langle f^{(1)}(x)\right\rangle\right\rangle f^{\left(2^{\prime}\right)}(A)
$$

where $f(x A) \equiv f^{(1)}(x) f^{\left(2^{\prime}\right)}(A)$. Consider now the dual action of the generators $Y_{i j} \equiv$ $L_{i m}^{+} S\left(L_{m j}^{-}\right)$of $\mathcal{U}_{q}(g l(N))$ on the integrand

$$
\left\langle\left\langle Y_{i j} \triangleright f\right\rangle\right\rangle=\left\langle\left\langle f^{(1)}\right\rangle\right\rangle\left\langle Y_{i j}, f^{\left(2^{\prime}\right)}\right\rangle .
$$

The above action can be represented in terms of differential operators on the plane as follows [4, 5]

$$
Y_{i j} \sim q^{-2} \delta_{i j}+q^{-1} \lambda \partial_{i} x_{j}
$$

which gives, making use of the invariance of the integral

$$
\begin{aligned}
\left\langle\left\langle Y_{i j} \triangleright f\right\rangle\right\rangle & =\left\langle\left\langle\left(q^{-2} \delta_{i j}+q^{-1} \lambda \partial_{i} x_{j}\right) f\right\rangle\right\rangle \\
& =q^{-2} \delta_{i j}\langle\langle f\rangle\rangle .
\end{aligned}
$$

Repeating the calculation for products of $Y^{\prime}$ 's acting on $f$, we find

$$
\left\langle\left\langle f^{(1)}\right\rangle\right\rangle \otimes f^{\left(2^{\prime}\right)}=\langle\langle f\rangle\rangle \otimes z
$$

with $\Delta(z)=z \otimes z$ and $\left\langle Y_{i j}, z\right\rangle=q^{-2} \delta_{i j},\left\langle 1_{\mathcal{U}}, z\right\rangle=1$. The above information completely determines $z$. Since $\left\langle Y_{i j}, \operatorname{det}_{\mathrm{q}}(A)\right\rangle=q^{2}, \epsilon\left(\operatorname{det}_{\mathrm{q}}(A)\right)=1$ and $\operatorname{det}_{\mathrm{q}}(A)$ is grouplike, we conclude

$$
\langle\langle f(x A)\rangle\rangle=\langle\langle f(x)\rangle\rangle\left(\operatorname{det}_{\mathrm{q}}(A)\right)^{-1} .
$$

As in the case of the quantum superplane, to obtain the correct $q$ factors for the braiding (so that, for example, $x \rightarrow x \underline{\otimes}+\underline{\underline{\otimes}} x$ is a homomorphism of the first of (108)) one has to introduce a grouplike, central dilaton $g$ (extending this way $\mathcal{A}$ to $\tilde{\mathcal{A}}$ ) with

$$
\left\langle\mathcal{R}, g^{a} \otimes g^{b}\right\rangle=q^{a b}, \quad\left\langle\mathcal{R}, g^{a} \otimes A_{i j}\right\rangle=\left\langle\mathcal{R}, A_{i j} \otimes g^{a}\right\rangle=\delta_{i j}
$$


and use the $\tilde{\mathcal{A}}$ coaction $x \rightarrow x A g, \partial \rightarrow \partial S\left(A^{T}\right) g^{-1}$ in $(73)$. We obtain in this way the braiding relations

$$
\begin{aligned}
\Psi\left(\langle\langle f(x)\rangle\rangle \otimes x_{i}\right) & =q^{-(N+1)} x_{i} \otimes\langle\langle f(x)\rangle\rangle \\
\Psi\left(\langle\langle f(x)\rangle\rangle \otimes \partial_{i}\right) & =q^{N+1} \partial_{i} \otimes\langle\langle f(x)\rangle\rangle \\
\Psi(\langle\langle f(x)\rangle\rangle \otimes\langle\langle g(x)\rangle\rangle) & =q^{N(N+1)}\langle\langle g(x)\rangle\rangle \otimes\langle\langle f(x)\rangle\rangle .
\end{aligned}
$$

We point out that a translationally invariant integral on the quantum plane cannot be also invariant under the coacting quantum group - an assumption to the contrary is made in [10].

\section{$5 \quad$ Integration on Quantum Group Modules}

We present here an approach to integration on quantum spaces that are covariant under a quantum group transformation, which does not rely on the braided Hopf algebra structure we have assumed so far. The only necessary ingredients are

- a coaction $\Delta_{\mathcal{A}}: \mathcal{X} \rightarrow \mathcal{X} \otimes \mathcal{A}$, where $\mathcal{A}, \mathcal{X}$ are the algebras of functions on the quantum group and the quantum space respectively

- a map $\eta: \mathcal{X} \rightarrow C$ that respects the algebra structure of $\mathcal{X}$

- a (left) invariant integral on $\mathcal{A}$.

Given the above data, an invariant (under $\Delta_{\mathcal{A}}$ ) integral on $\mathcal{X}$ can be defined by

$$
\langle\alpha\rangle=\eta\left(\alpha^{(1)}\right)\left\langle\alpha^{\left(2^{\prime}\right)}\right\rangle
$$

(we denote by $\langle\cdot\rangle$ the integral on both $\mathcal{X}$ and $\mathcal{A}$ ). Notice that our notion of invariance in this section is different from the one employed so far in this paper. Indeed, in the absence of a (possibly braided) Hopf structure, no concept of translation exists (as codified by the coproduct) and therefore (10) cannot serve as our starting point. We illustrate the above procedure taking for $\mathcal{X}$ the quantum Euclidean space (for detailed treatments of this case see [8, 21]). An example of a function algebra the integral on which eludes all methods presented so far in this paper appears in [6]).

\subsection{Integration on the quantum Euclidean space}

In the following we use the notation and conventions of [9]. The algebra of functions on the $N$-dimensional quantum Euclidean space is generated by the coordinates $x_{i}, i=1, \ldots, N$ satisfying

$$
x_{1} x_{2} P_{12}^{(-)}=0
$$

where $P^{(-)}$is the antisymmetric projector in the spectral decomposition of the $S O_{q}(N) \hat{R}$ matrix. The center of the algebra is generated by 1 and the squared length $L^{2}=x^{T} C x$ where $C$ is the quantum metric. For $A$ an $S O_{q}(N)$ matrix, it holds

$$
S^{2}(A)=D A D^{-1}, \quad D=C C^{T}, \quad A=C^{T} S\left(A^{T}\right)\left(C^{-1}\right)^{T} .
$$

The algebra of the $x$ 's admits the coaction $\Delta_{\mathcal{A}}: x \mapsto x A$ while the map $\eta: x_{i} \mapsto u_{i} \equiv$ $u_{1} \delta_{i 1}+u_{N} \delta_{i N}$, with $u_{1}, u_{N}$ numbers, respects (118). $L^{2}$ is $\Delta_{\mathcal{A}}$-invariant. 
The integral $\mathcal{I}_{i_{1} \ldots i_{m}} \equiv\left\langle x_{i_{1}} \ldots x_{i_{m}}\right\rangle$ is given by

$$
\mathcal{I}_{i_{1} \ldots i_{m}}=\eta\left(x_{j_{1}} \ldots x_{j_{m}}\right)\left\langle A_{j_{1} i_{1}} \ldots A_{j_{m} i_{m}}\right\rangle
$$

Notice that the function $x_{j_{1}} \ldots x_{j_{m}}\left\langle A_{j_{1} i_{1}} \ldots A_{j_{m} i_{m}}\right\rangle$ is $\Delta_{\mathcal{A}^{-}}$-invariant. Assuming that all $\Delta_{\mathcal{A}^{-}}$ invariant functions are functions of the invariant length, we conclude

$$
x_{j_{1}} \ldots x_{j_{m}}\left\langle A_{j_{1} i_{1}} \ldots A_{j_{m} i_{m}}\right\rangle=\left\{\begin{array}{cl}
f_{i_{1} \ldots i_{m}}\left(L^{m}\right) & m \text { even } \\
0 & m \text { odd }
\end{array}\right.
$$

and therefore

$$
\mathcal{I}_{i_{1} \ldots i_{m}}=\left\{\begin{array}{cl}
\eta\left(f_{i_{1} \ldots i_{m}}\left(L^{2}\right)\right) & m \text { even } \\
0 & m \text { odd }
\end{array} .\right.
$$

We treat, as an example, the case $m=2$. Setting

$$
F_{n i, m j} \equiv\left\langle S\left(A_{i m}\right) A_{n j}\right\rangle
$$

and invoking invariance in the form $S\left(a_{(1)}\right)\left\langle a_{(2)}\right\rangle=1\langle a\rangle$, we get

$$
\begin{aligned}
A_{r b} F_{b i, a j} & =A_{r b} S\left(A_{b m}\right) S^{2}\left(A_{n i}\right) F_{m a, n j} \\
\Rightarrow A_{1} F_{12} D_{1} & =F_{12} D_{1} A_{1} .
\end{aligned}
$$

Since only multiples of the identity matrix commute with $A$, we conclude

$$
F_{12}=M_{2} D_{1}^{-1}
$$

However,

$$
\begin{aligned}
A_{k i} F_{b i, a j} & =A_{k i} S\left(A_{i n}\right) A_{r j} F_{b n, a r} \\
\Rightarrow A_{2} F_{12} & =F_{12} A_{2} \\
\Rightarrow M_{2} & =\rho I_{2}
\end{aligned}
$$

with $\rho$ a number. With the normalization $\left\langle 1_{\mathcal{A}}\right\rangle=1$, we find $\rho=\left(\operatorname{Tr} D^{-1}\right)^{-1}$ and therefore

$$
F_{b i, a j}=\frac{1}{\operatorname{Tr} D^{-1}} D_{b a}^{-1} \delta_{i j}
$$

Using now the third of (119) in (120) and substituting the above result, we find, with the help of (119),

$$
\mathcal{I}_{i_{1} i_{2}}=\frac{\eta\left(L^{2}\right)}{\operatorname{Tr} D^{-1}} C_{i_{1} i_{2}}
$$

\section{Acknowledgements}

Many people have helped me understand the subject matter treated here. I thank Joris Van der Jeught for discussions and computations that convinced me to treat the infinite dimensional case elsewhere. While this paper was being written up, A. Van Daele kindly pointed out reference [23] which contains essentially (23). Also, the alternative proof of Lemma 1 quoted here (ending in eqn. (25)) motivated the proof of Lemma 6 - I thank him for his comments during the discussions we had in Warsaw. I also thank for discussions Shahn Majid 
and Volodimir Lyubashenko (who also drew my attention to references [13, 14]). Thanks go to Daniel Arnaudon for his help with all things binary. Special thanks go to Stanislaw Zakrzewski for his interest in this paper and for the organization of (and invitation to) the minisemester on Quantum Groups and Quantum Spaces (November - December 1995, Warsaw) during which many aspects of the subject treated here were illuminated. Bruno Zumino had a decisive impact on this work through numerous discussions, contributions and his constant support my warmest thanks go to him.

\section{References}

[1] Abe E., "Hopf Algebras", Cambridge Univ. Press, 1980

[2] Chryssomalakos C., Schupp P., Watts P., "The Role of the Canonical Element in the Quantized Algebra of Differential Operators $\mathcal{A} \rtimes \mathcal{U}^{\prime \prime}$, preprint LBL-33274, UCB-PTH$92 / 42$

[3] Chryssomalakos, Zumino B., "Translations, Integrals And Fourier Transforms In The Quantum Plane" in A. Ali, J. Ellis and S. Randjbar-Daemi, eds., "Salamfestschrift", proceedings of the "Conference On Highlights Of Particle And Condensed Matter Physics", ICTP, Trieste, Italy (1993)

[4] Chryssomalakos C., Schupp S., Zumino B., "Induced Extended Calculus On The Quantum Plane", Alg. i Anal. 6252 (1994)

[5] Chu C. S., Zumino B., "Realization of Vector Fields for Quantum Groups as Pseudodifferential Operators on Quantum Spaces", preprint LBL-36746, UCB-PTH-95/04, q-alg/9502005

[6] Chu C.-S., Ho P.-M., Zumino B., "The Quantum 2-Sphere as a Complex Quantum Manifold", to appear in Z. Phys. C

[7] Drinfel'd V. G., "Quantum Groups", in A. Gleason (ed.), Proceedings of the ICM, Rhode Island (1987), AMS

[8] Fiore G., "The $S O_{q}(N, \mathbf{R})$-Symmetric Harmonic Oscillator on the Quantum Euclidean Space $\mathbf{R}_{q}^{N}$ and its Hilbert Space Structure", Int. J. Mod. Phys. A8, 4679-4729 (1993)

[9] Faddeev L. D., Reshetikhin N. Yu. and Takhtadzhyan L. A., "Quantization of Lie Groups and Lie Algebras", Leningrad Math. J. 1 193-225 (1990)

[10] Kempf A., Majid S., "Algebraic q-integration and Fourier Theory on Quantum and Braided Spaces", preprint DAMTP/94-7

[11] Larson R. G., Radford D. E., "Semisimple Cosemisimple Hopf Algebras" Am. J. Math. 109187 (1988)

[12] Larson R.G., Sweedler M.E., "An Associative Orthogonal Bilinear Form for Hopf Algebras", Amer. J. Math. 91 75-94 (1969) 
[13] Lyubashenko V., "Tangles and Hopf Algebras In Braided Categories", J. Pur. Appl. Alg 98 245-278 (1995)

[14] Lyubashenko V., "Modular Transformations for Tensor Categories", J. Pur. Appl. Alg. 98 279-327 (1995)

[15] Majid S., "Quasitriangular Hopf Algebras and Yang-Baxter Equations", Int. J. Mod. Phys. A5 1-91 (1990)

[16] Majid S., "Braided Momentum in the q-Poincare Group", J. Math. Phys. 34 2045-2058 (1993)

[17] Majid S., "Beyond Supersymmetry And Quantum Symmetry (an introduction to braided groups and braided matrices)", in Ge M. L., de Vega H. J., eds., "Quantum Groups, Integrable Statistical Models and Knot Theory", pp. 231-282, Wld. Sci. (1993)

[18] Majid S., "Lie Algebras And Braided Geometry", Adv. Appl. Cliff. Alg. 4 (S1) 61-77 (1994)

[19] Radford D.E., "The Trace Function And Hopf Algebras", preprint

[20] Radford D. E., "The Order of the Antipode of a Finite Dimensional Hopf Algebra is Finite", Am. J. Math. 98 (2) 333-355 (1973)

[21] Steinacker H., "Integration on Quantum Euclidean Space and Sphere in N Dimensions", preprint LBL-37431, UCB-PTH-95/21; q-alg 9506020

[22] Sweedler M. E., "Hopf Algebras", Benjamin (1969)

[23] Van Daele A., "The Haar Measure On Finite Quantum Groups", preprint K. U. Leuven, November 1992.

[24] Van Daele A., private communication

[25] Wess J., Zumino B., "Covariant Differential Calculus on the Quantum Hyperplane", Nucl. Phys. B (Proc. Suppl.) 18B 302-312 (1990)

[26] Zumino B., private communication

[27] Zumino B., "Deformation of the Quantum Mechanical Phase Space with Bosonic or Fermionic Coordinates", Mod. Phys. Lett. A 13 1225-1235 (1991) 